\title{
Isolation and sequence-based characterization of a koala symbiont: Lonepinella koalarum
}

\author{
Katherine E Dahlhausen ${ }^{1}$, Guillaume Jospin ${ }^{1}$, David A Coil ${ }^{1}$, Jonathan A Eisen ${ }^{1,2,3}$, Laetitia G E Wilkins ${ }^{\text {Corresp. } 1}$ \\ ${ }^{1}$ Genome and Biomedical Sciences Facility, University of California, Davis, Davis, California, United States \\ 2 Department of Evolution and Ecology, University of California, Davis, Davis, California, United States \\ 3 Department of Medical Microbiology and Immunology, University of California, Davis, Davis, California, United States \\ Corresponding Author: Laetitia G E Wilkins \\ Email address: megaptera.helvetiae@gmail.com
}

Koalas (Phascolarctos cinereus) are highly specialized herbivorous marsupials that feed almost exclusively on Eucalyptus leaves, which are known to contain varying concentrations of many different toxic chemical compounds. The literature suggests that Lonepinella koalarum, a bacterium in the Pasteurellaceae family, can break down some of these toxic chemical compounds. Furthermore, in a previous study, we identified $L$. koalarum as the most predictive taxon of koala survival during antibiotic treatment. Therefore, we believe that this bacterium may be important for koala health. Here, we isolated a strain of $L$. koalarum from a healthy koala female and sequenced its genome using a combination of short-read and long-read sequencing. We placed the genome assembly into a phylogenetic tree based on 120 genome markers using the Genome Taxonomy Database (GTDB), which currently does not include any L. koalarum assemblies. Our genome assembly fell in the middle of a group of Haemophilus, Pasteurella and Basfia species. According to average nucleotide identity and a 16S rRNA gene tree, the closest relative of our isolate is $L$. koalarum strain Y17189. Then, we annotated the gene sequences and compared them to 55 closely related, publicly available genomes. Several genes that are known to be involved in carbohydrate metabolism could exclusively be found in $L$. koalarum relative to the other taxa in the pangenome, including glycoside hydrolase families GH2, GH31, GH32, GH43 and GH77. Among the predicted genes of $L$. koalarum were 79 candidates putatively involved in the degradation of plant secondary metabolites. Additionally, several genes coding for amino acid variants were found that had been shown to confer antibiotic resistance in other bacterial species against pulvomycin, beta-lactam antibiotics and the antibiotic efflux pump $\mathrm{KpnH}$. In summary, this genetic characterization allows us to build hypotheses to explore the potentially beneficial role that $L$. koalarum might play in the koala intestinal microbiome. Characterizing and understanding beneficial symbionts at the whole genome level is important for the 
development of anti- and probiotic treatments for koalas, a highly threatened species due to habitat loss, wildfires, and high prevalence of Chlamydia infections. 


\section{Isolation and sequence-based characterization of a koala symbiont: \\ 2 Lonepinella koalarum}

3

4 Katherine E. Dahlhausen ${ }^{1}$, Guillaume Jospin ${ }^{1}$, David A. Coil ${ }^{1}$, Jonathan A. Eisen ${ }^{1,2,3}$, and

5 Laetitia G. E. Wilkins ${ }^{1}$

6

$7{ }^{1}$ Genome and Biomedical Sciences Facility, University of California, Davis, Davis, CA 95616, 8 USA

92 Department of Evolution and Ecology, University of California, Davis, Davis, CA 95616, USA

$10{ }^{3}$ Department of Medical Microbiology and Immunology, University of California, Davis, Davis, 11 CA 95616, USA

13 Corresponding Author:

14 Laetitia Wilkins ${ }^{1}$

15451 Health Science Drive

16 Genome and Biomedical Sciences Facility

17 University of California, Davis

18 Davis, CA, 95616, United States

19 Email address: megaptera.helvetiae@gmail.com 
20 Abstract

21 Koalas (Phascolarctos cinereus) are highly specialized herbivorous marsupials that feed almost

22 exclusively on Eucalyptus leaves, which are known to contain varying concentrations of many

23 different toxic chemical compounds. The literature suggests that Lonepinella koalarum, a

24 bacterium in the Pasteurellaceae family, can break down some of these toxic chemical

25 compounds. Furthermore, in a previous study, we identified L. koalarum as the most predictive

26 taxon of koala survival during antibiotic treatment. Therefore, we believe that this bacterium

27 may be important for koala health. Here, we isolated a strain of L. koalarum from a healthy koala

28 female and sequenced its genome using a combination of short-read and long-read sequencing.

29 We placed the genome assembly into a phylogenetic tree based on 120 genome markers using

30 the Genome Taxonomy Database (GTDB), which currently does not include any L. koalarum

31 assemblies. Our genome assembly fell in the middle of a group of Haemophilus, Pasteurella and

32 Basfia species. According to average nucleotide identity and a 16S rRNA gene tree, the closest

33 relative of our isolate is L. koalarum strain Y17189. Then, we annotated the gene sequences and

34 compared them to 55 closely related, publicly available genomes. Several genes that are known

35 to be involved in carbohydrate metabolism could exclusively be found in L. koalarum relative to

36 the other taxa in the pangenome, including glycoside hydrolase families GH2, GH31, GH32,

37 GH43 and GH77. Among the predicted genes of L. koalarum were 79 candidates putatively

38 involved in the degradation of plant secondary metabolites. Additionally, several genes coding

39 for amino acid variants were found that had been shown to confer antibiotic resistance in other

40 bacterial species against pulvomycin, beta-lactam antibiotics and the antibiotic efflux pump

$41 \mathrm{KpnH}$. In summary, this genetic characterization allows us to build hypotheses to explore the

42 potentially beneficial role that L. koalarum might play in the koala intestinal microbiome.

43 Characterizing and understanding beneficial symbionts at the whole genome level is important

44 for the development of anti- and probiotic treatments for koalas, a highly threatened species due

45 to habitat loss, wildfires, and high prevalence of Chlamydia infections.

\section{Introduction}

48 Koalas (Phascolarctos cinereus) are arboreal marsupials that are highly specialized herbivores in

49 that they feed almost exclusively on the foliage of select Eucalyptus species (Moore \& Foley,

50 2005; Callaghan et al., 2011). All Eucalyptus species contain chemical defenses against 
51 herbivory that include tannins, B-ring flavanones, phenolic compounds, terpenes, formylated 52 phloroglucinols, cyanogenic glucosides, and other plant secondary metabolites (Lawler, Foley \& 53 Eschler, 2000; Gleadow et al., 2008; Brice et al., 2019; Liu et al., 2019). Plant chemical defenses 54 can deter herbivores by affecting taste and/or digestibility of ingested material, with varying 55 levels of toxic effects (Brice et al., 2019). These defenses and anti-nutrient compounds, which 56 will be referred to generally as 'PCDs' (for plant chemical defenses) hereafter, are very common, and there is well-established evidence that herbivores can overcome these defenses in their diet at least in part via PCD-degradation by their intestinal microbial communities (Freeland \& Janzen, 1974; Waterman et al., 1980; Hammer \& Bowers, 2015; Kohl \& Denise Dearing, 2016). However, it is unknown to what extent Eucalyptus PCDs are degraded by the intestinal microbial communities of koalas.

Research has highlighted several ways in which koalas are able to manage such a toxic diet independent of the functions of their intestinal microbial communities. For example, some studies suggest that koalas can minimize PCDs intake through tree- and even leaf selection (Lawler, Foley \& Eschler, 2000; Marsh et al., 2003; Moore \& Foley, 2005; Liu et al., 2019). Another study identified several genes in the koala genome that are associated with metabolism and detoxification of many types of xenobiotics (Johnson et al., 2018). The findings from a study on microsomal samples from koala liver also suggest that koalas are able to metabolize some xenobiotics in their livers (Ngo et al., 2000). Furthermore, other research suggests that toxic compounds found in Eucalyptus may be absorbed in the upper digestive system before they even reach the intestinal microbial community of herbivores (Foley, Lassak \& Brophy, 1987).

The intestinal microbial communities of koalas are also thought to contribute to the management and degradation of PCDs found in Eucalyptus leaves. To date, the most compelling evidence for this is included in a recent study on the koala fecal microbiome, which identified several metabolic pathways and relevant bacterial species proposed to be important in detoxification processes (Shiffman et al., 2017). The koala microbiome as a whole has shown to play an important role in macro nutrient digestion and fiber degradation (Blyton et al., 2019; Brice et al., 2019). Moreover, there is evidence that the koala gastrointestinal microbiome can influence diet selection of individual hosts (Blyton et al., 2019). At the individual level, several bacterial 
82 isolates associated with the intestinal microbial communities of koalas have been characterized 83 in the context of degradation of PCDs found in Eucalyptus leaves (Osawa, 1990, 1992; Osawa et 84 al., 1993, 1995; Looft, Levine \& Stanton, 2013). One of these cultured isolates is a bacterium 85 known as Lonepinella koalarum. It had been first isolated from the mucus around the caecum in 86 koalas and was shown to degrade tannin-protein complexes that can be found in Eucalyptus 87 leaves (Osawa et al., 1995; Goel et al., 2005). Briefly, tannin-protein complexes are extremely 88 diverse and result from the reaction between plant defense secondary metabolites; i.e., tannins, 89 and proteins. Tannins bind proteins followed by the formation of a precipitate, which cannot be 90 digested by koalas or utilized by microbes (Adamczyk et al., 2017). In our previous work, $L$. 91 koalarum was identified as the most predictive taxon of koala survival during antibiotic 92 treatment (Dahlhausen et al., 2018). Briefly, a co-occurrence network analysis identified four 93 bacterial taxa, including one of the genus Lonepinella, that could be found in feces of koalas that survived their antibiotic treatment after Chlamydia infection. However, these four taxa were absent from feces of koalas that died. Furthermore, in the same study a random forest analysis revealed that the most predictive taxon of whether a koala would live or die during their antibiotic treatment was identified as L. koalarum. This finding suggests that L. koalarum could be important for koala health, but the study did not present any evidence relating to PCD degradation in the highly specialized diet of koalas.

100

It is well understood that animals with highly specialized diets also are likely to have highly specialized intestinal microbial communities (Higgins et al., 2011; Kohl et al., 2014; Alfano et al., 2015; Kohl, Stengel \& Denise Dearing, 2016). Disturbances of a specialized microbial community, such as the introduction of antibiotics, can have profound effects on the host's health (Kohl \& Denise Dearing, 2016; Brice et al., 2019). Yet, koalas are regularly treated with antibiotics due to the high prevalence of Chlamydia infections in many populations (Polkinghorne, Hanger \& Timms, 2013). While recent advances in Chlamydia pecorum vaccines for koalas are a promising alternative for managing koala populations, antibiotics are still the current treatment method for bacterial infections in koalas (Waugh et al., 2016; Desclozeaux et al., 2017; Nyari et al., 2018). The antibiotics used in practice might not only target Chlamydia pecorum but also beneficial koala gut symbionts as a side effect. Therefore, it is important to learn about bacteria associated with koala health, such as L. koalarum, in order to further the 
113 development of alternative treatments for bacterial infections in koalas and to recommend

114 antibiotic compounds that are potentially less disruptive to members of the koala gut

115 microbiome.

116

117 Here we isolated a strain of L. koalarum (hereafter called strain UCD-LQP1) from the feces of a

118 healthy koala (P. cinereus) female at the San Francisco Zoo. We sequenced the genome of $L$.

119 koalarum UCD-LQP1 using a combination of long- and short-read sequencing, and then

120 assembled and annotated the genome. We compared the genome assembly to the most closely

121 related genomes that are currently publicly available. The genome assembly of L. koalarum

122 UCD-LQP1 was placed in a phylogenetic tree and screened for genes putatively involved in the

123 degradation of plant secondary metabolites, carbohydrate metabolism, and antibiotic resistance.

124 Additionally, we identified and characterized putative genes that were unique to this strain and

125 two recently sequenced genomes of $L$. koalarum from Australia.

126

127 Materials and methods

128 Sampling of koala feces and preparation of culturing media

129 A koala fecal pellet was collected, with permission from the San Francisco Zoo, from a healthy, 130 adult, captive, female koala (Phascolarctos cinereus). We do not have any information on the 131 geographical origin of this koala. Koalas at the SF Zoo are fed blue gum leaves (Eucalyptus 132 globulus), which grow quite abundantly in California. Jim Nappi and Graham Crawford of the

133 San Francisco Zoo organized and permitted koala fecal sample collection. The fresh fecal pellet 134 was collected from the floor with sterilized tweezers and stored in a sterile $15 \mathrm{ml}$ Falcon tube 135 (Thermo Fisher Scientific, USA). The tube was immediately placed on ice after collection and 136 subsequently stored at $4^{\circ} \mathrm{C}$ overnight.

137

138 The preparation of the Lonepinella koalarum culturing media was modified from methods 139 developed by Osawa et al. (1995). A $2 \%$ agarose (Fisher BioReagents, USA) solution of 140 Bacto $^{\mathrm{TM}}$ Brain Heart Infusion (BHI; BD Biosciences, USA) was prepared following

141 manufacturer protocols. After the media had solidified in petri dishes, a $2 \%$ tannic acid solution 142 was prepared by combining $1 \mathrm{~g}$ of tannic acid powder per $50 \mathrm{ml}$ of sterile Nanopure ${ }^{\mathrm{TM}}$ water 143 (Spectrum Chemical MFG CORP, USA). The solution was vortexed for 1 min until 
144 homogenized, resulting in a brown, transparent liquid. Using a sterile serological pipette, $5 \mathrm{ml}$ of 145 the $2 \%$ tannic acid solution was gently added to each BHI media plate and left for 20 min. After 146 incubation, the remaining liquid on the plate was decanted. No antibiotic compounds were added 147 to the medium.

\section{Culturing of isolates and DNA extraction}

149 The koala fecal pellet was cut in half with sterile tweezers. Tweezers were re-sterilized and used 150 to move approximately $300 \mathrm{mg}$ of material from the center of the pellet to a sterile $2 \mathrm{ml}$

151 Eppendorf tube containing $1 \mathrm{ml}$ of sterile, Nanopure ${ }^{\mathrm{TM}}$ water. The tube was vortexed for $3 \mathrm{~min}$, 152 intermittently checking until the solution was homogenized into a slurry. One hundred $\mu 1$ of the 153 homogenized fecal slurry was micro-pipetted onto to a BHI+tannin plate and stored in an 154 anaerobic chamber (BD GasPak ${ }^{\mathrm{TM}} \mathrm{EZ}$ anaerobe chamber system; BD Biosciences, USA) at $37^{\circ}$

$155 \mathrm{C}$ for 3 days. Each individual colony that grew was plated onto a freshly made BHI+tannin plate 156 using standard dilution streaking techniques. The new plates were stored in an anaerobic 157 chamber at $37^{\circ} \mathrm{C}$ for another 3 days. This step was repeated two more times to decrease the 158 probability of contamination or co-culture.

159

160 An individual colony from each of the plates from the third round of dilution streaking was 161 moved to a sterile $30 \mathrm{ml}$ glass culture tube containing $5 \mathrm{ml}$ of sterile Bacto ${ }^{\mathrm{TM}}$ BHI liquid media 162 (prepared following manufacturer protocol; BD Biosciences, USA). Each tube was then capped 163 with a sterile rubber stopper and purged with nitrogen gas in order to create an anaerobic 164 environment. The tubes were placed in an incubated orbital shaker (ThermoFisher Scientific $165 \mathrm{MaxQ}^{\mathrm{TM}} 4450$ ) for 3 days at $37^{\circ} \mathrm{C}$ at $250 \mathrm{rpm}$.

166

167 Using a sterile serological pipette, $1.8 \mathrm{ml}$ of each liquid culture was transferred to a sterile $2 \mathrm{ml}$ 168 Eppendorf tube. The tubes were spun at $13,000 \mathrm{~g}$ for $2 \mathrm{~min}$ and the supernatant was carefully 169 decanted. The DNA was extracted from the pellet in each sample with the Promega Wizard 170 Genomic DNA Purification Kit (Promega, USA) according to the manufacturer's protocol. DNA 171 was eluted in a final volume of $100 \mu \mathrm{l}$ and stored at $4{ }^{\circ} \mathrm{C}$. 
$172 \quad P C R$ and Sanger sequencing

173 PCR amplification of the 16S rRNA gene was performed on each of the eluted DNA samples.

174 PCR reactions were prepared using the bacteria-specific "universal" primer pair 27F (5'-

175 AGAGTTTGATCMTGGCTCAG-3'; Stackebrandt \& Goodfellow, 1991) and 1391R (5'-

176 GACGGGCGGTGTGTRCA-3'; Turner et al., 1999). PCR amplifications were performed in a

177 BioRad T100 ${ }^{\mathrm{TM}}$ Thermal Cycler in $50 \mu \mathrm{l}$ reactions. Each reaction contained $2 \mu \mathrm{l}$ of the eluted

178 DNA from the aforementioned extraction, $5 \mu 1$ of 10x Taq buffer (Qiagen, USA), $10 \mu 1$ of Q

179 buffer (Qiagen), $1.25 \mu \mathrm{l}$ of 10mM dNTPs (Qiagen), $2.5 \mu 1$ of 10mM 27F primer, $2.5 \mu 1$ of 10mM

180 1391R primer, $0.3 \mu \mathrm{l}$ of Taq polymerase (Qiagen), and $26.45 \mu \mathrm{l}$ of sterile water. The cycling

181 conditions were: (1) $95{ }^{\circ} \mathrm{C}$ for $3 \mathrm{~min}$, (2) 40 cycles of $15 \mathrm{sec}$ at $95{ }^{\circ} \mathrm{C}, 30 \mathrm{sec}$ at $54{ }^{\circ} \mathrm{C}$, and $1 \mathrm{~min}$

182 at $72{ }^{\circ} \mathrm{C}$, (3) a final incubation at $72{ }^{\circ} \mathrm{C}$ for $5 \mathrm{~min}$, and (4) holding at $12{ }^{\circ} \mathrm{C}$ upon completion. 183

184 The PCR product for each sample was purified and concentrated by following the

185 manufacturer's protocol for the NucleoSpin Gel and PCR Clean-up kit (Macherey-Nagel, USA).

186 The purified PCR product for each sample was quantified by following the manufacturer's

187 protocol for the Qubit dsDNA HS Assay Kit (Thermo Fisher Scientific, USA). The PCR product

188 for each sample was then diluted to $26 \mathrm{ng} / \mu 1$ and submitted for forward and reverse Sanger

189 sequencing at the University of California Davis DNA Sequencing Facility. The program

190 SeqTrace version 0.9.0 (Stucky, 2012) was used to edit and create consensus sequences of the

191 reads received from the sequencing facility, following the protocol detailed in Dunitz et al.

192 (2015). The consensus sequence for each sample was uploaded to the NCBI blast website for

193 organism identification (Madden, 2003). The DNA of one of the isolates that had been identified

194 as L. koalarum was used for whole-genome sequencing, as described below. We refer to this

195 isolate as L. koalarum strain UCD-LQP1.

196

197

198

199

200

201

\section{Whole genome sequencing and assembly}

DNA from one sample identified as L. koalarum strain UCD-LQP1 was submitted for whole genome PacBio sequencing at SNPsaurus. After sequencing, the demultiplexed bam file was tested for reads that contained palindromic sequences since a preliminary assembly with Canu version 1.8 (Koren et al., 2017) indicated the presence of adapter sequences. Palindromic reads were split in half, aligned with minimap2 (an executable in Canu), and those palindromic reads 
202 that aligned over at least two-thirds of the split read were reduced to the first part of the 203 palindrome (Koren et al., 2017). This procedure efficiently removed adapter sequences. These 204 adapter-free reads were used in the hybrid assembly described below.

205

206 The same DNA that had been used for PacBio sequencing was also submitted for Illumina 207 sequencing. Ten ng of genomic DNA were used in a 1:10 reaction of the Nextera DNA Flex 208 Library preparation protocol (Illumina, USA). Fragmented DNA was amplified with Phusion 209 DNA polymerase (New England Biolabs) in 12 PCR cycles with 1 min extension time. Samples 210 were sequenced on a HiSeq4000 instrument (University of Oregon GC3F) with paired-end 150

211 bp reads. The 10,309,488 raw reads were quality controlled and filtered for adaptors and PhiX 212 using the Joint Genome Institute's BBDuk tool version 37.68 (Bushnell, 2014), resulting in 213 10,302,312 reads. The 308 cleaned PacBio reads and 10,302,312 filtered Illumina reads were 214 combined with all default parameters of Unicycler version 0.4 .5 , a tool used to assemble 215 bacterial genomes from both long and short reads (Wick et al., 2017).

\section{Genome annotation}

217 Completeness and contamination of the L. koalarum strain UCD-LQP1 assembly were 218 determined with CheckM version 1.0.8 (Parks et al., 2015), number of contigs, total length, 219 GC\%, N50, N75, L50, and L75 were determined with QUAST (Quality Assessment Tool for 220 Genome Assemblies; Gurevich et al., 2013), and the assembly was annotated with PROKKA 221 version 1.12 (Seemann, 2014). The L. koalarum strain UCD-LQP1 genome assembly was 222 uploaded to the Rapid Annotation using Subsystem Technology online tool (RAST), a genome 223 annotation program for bacterial and archaeal genomes (Aziz et al., 2008). The SEED viewer in 224 RAST was used to browse features of the genome (Overbeek et al., 2014). To screen the $L$. 225 koalarum strain UCD-LQP1 assembly for genes putatively involved in tannin degradation and 226 xenobiotic metabolisms; i.e., the degradation of plant secondary metabolites, coding regions in 227 the assembly were identified using Prodigal version 2.6.3 (Hyatt et al., 2010). Each identified 228 coding region was annotated using eggNOG (a database of orthologous groups and functional 229 annotation that is updated more regularly than PROKKA) mapper version 4.5.1 (Jensen et al., 230 2008). Then, KEGG (Kyoto Encyclopedia of Genes and Genomes) pathways putatively involved 231 in xenobiotics biodegradation and metabolism, were extracted from the eggNOG annotations 
232 (Class 1.11 Xenobiotics biodegradation and metabolism includes the following KEGG pathways: 233 ko00362, ko00627, ko00364, ko00625, ko00361, ko00623, ko00622, ko00633, ko00642, 234 ko00643, ko00791, ko00930, ko00363, ko00621, ko00626, ko00624, ko00365, ko00984, 235 ko00980, ko00982, and ko00983 (Kanehisa \& Goto, 2000)), and the corresponding nucleotide 236 sequences from the L. koalarum genome assemblies were saved. Individual genes with hits in 237 KEGG pathways were manually mapped onto KEGG reference maps using the KEGG webtool 238 (Kanehisa \& Goto, 2000).

239

240 241

242 243 244 245 246 247 248 249 250 251 252 253 254 255 256 257 258

\section{$16 S$ rRNA gene based phylogenetic placement of genome}

The 16S rRNA gene sequence within the genome assembly was extracted from RAST by searching for 'ssu rRNA' in the function search of the SEED genome browser (Aziz et al., 2008; Overbeek et al., 2014). Following the protocol outlined in Dunitz et al. (2015), the 16S rRNA gene sequence was uploaded to the Ribosomal Database Project (RDP; Cole et al., 2014) and grouped with all 16S rRNA gene sequences in the Pasteurellaceae family and one chosen outgroup, Agarivoran spp., to root the tree. The taxon names from the RDP output file were manually cleaned up and their 16S rRNA gene sequences were used to build a phylogenetic tree with the program FastTree (Price, Dehal \& Arkin, 2009). Nodes and tip labels were manually edited for Figure 1 in iTOL (interactive tree of life; web tool; Letunic \& Bork, 2019). The 16S rRNA gene sequence alignment (Wilkins \& Coil, 2020a) and its resulting phylogenetic tree are available on Figshare (Wilkins \& Coil, 2020b). During the preparation of this manuscript, two more L. koalarum type strains had their genomes sequenced: one by the DOE Joint Genome Institute, USA (GenBank accession number GCA_004339625.1; 2,486,773 bp long) and one by the Maclean Lab in Australia (GenBank accession number GCA_004565475.1; 2,509,358 bp). Both assemblies were based on type strains originating from the same isolation of L. koalarum in 1995 (Osawa et al., 1995), DSM 10053 and ATCC 700131, respectively. These two L. koalarum genome assemblies were henceforth included in our analysis. When we refer to all three $L$. koalarum genome assemblies, we simply say 'in L. koalarum' and when we refer to the strain sequenced in this study, we use 'the assembly of $L$. koalarum strain UCD-LQP1'. 
259 Comparative genomics

260 The GTDB-Tk software toolkit version 0.3.0 (Chaumeil, Hugenholtz \& Parks, 2018) of the

261 Genome Taxonomy Database (GTDB) project was chosen to place L. koalarum into a pre-

262 generated conserved marker gene tree using 120 marker genes (Parks et al., 2018). After placing

263 the assembly into the GTDB tree, a clade in the tree was extracted that contained L. koalarum

264 strain UCD-LQP1 and 55 other taxa, of which all members belonged to the order Pasteurellales.

265 This clade contained all sequenced genomes of the closest neighboring taxa $(\mathrm{n}=55)$ to $L$.

266 koalarum in the GTDB tree at the time of this analysis ( $3^{\text {rd }}$ of August 2019). All of these 55

267 genomes were downloaded from GenBank (using the accession numbers in the GTDB) to

268 perform a comparative genomic analysis in Anvi'o version 5.5 (Eren et al., 2015). The two other

269 L. koalarum genomes from GenBank were included in the following analysis as well. Accession

270 numbers of all genome assemblies included can be found in Supplementary Table S1 $(n=58)$.

271 The Anvi'o workflow for microbial pangenomics was followed (Delmont \& Eren, 2018). The

272 blastp program from NCBI was used for a gene search (Altschul et al., 1990), the Markov

273 Cluster algorithm (MCL) version 14.137 (van Dongen \& Abreu-Goodger, 2012) was used for

274 clustering, and the program MUSCLE was used for alignment (Edgar, 2004). An inflation

275 parameter of 6 was chosen to identify clusters in amino acid sequences. Genomes in the

276 pangenome of Anvi'o were ordered based on a genomic marker gene tree. This tree was built in

277 PhyloSift version 1.0.1 (Darling et al., 2014) with its updated markers database (version 4,

278 posted on 12th of February 2018; Jospin, 2018) for the alignment. We used RAxML version

279 8.2.10 on the CIPRES web server for the tree inference (Miller, Pfeiffer \& Schwartz, 2010)

280 following the analysis in (Wilkins et al., 2019). Gene clusters in Anvi'o were ordered based on

281 presence/absence. We also used Anvi'o to compute average nucleotide identities across the

282 genomes with PyANI (Pritchard et al., 2016). In the heatmap, ANI values $>95 \%$ (and $>70 \%$ for

283 a separate figure, respectively) were colored in red.

284

285 Gene clusters from the Anvi'o microbial pangenomics analysis that could only be found in the

286 three L. koalarum genome assemblies were extracted. Then, we also extracted all gene clusters

287 that could only be found in the assembly of L. koalarum strain UCD-LQP1. Partial sequences

288 were removed. A literature search of the remaining genes was conducted to identify possible 
289 roles L. koalarum might play in the gut microbiome of koalas. Tables were summarized in $\mathrm{R}$ 290 version 3.4.0 (R Development Core Team, 2013).

\section{Carbohydrate metabolism}

292 Since the majority of gene clusters unique to L. koalarum genome assemblies fell into the COG 293 (Clusters of Orthologous Groups) category 'Carbohydrate metabolism', we decided to screen all 294 three assemblies against the Carbohydrate-Active Enzymes Database (CAZy), an expert resource 295 for glycogenomics (Cantarel et al., 2009; Lombard et al., 2014). In brief, CAZy domains were 296 identified based on CAZy family HMMs (Hidden Markov Models) with a coverage of $>95 \%$ and 297 an e-value $<1$ e-15. Searches were done through dbCAN, a web resource for automated 298 carbohydrate-active enzyme annotation (Yin et al., 2012) and CAZy hits were only retained if they had been found with all three search tools. The three search tools included (i) HMMER version 3.3 (Eddy, 1998), (ii) DIAMOND version 0.9.29 for fast blast hits in the CAZy database (Buchfink, Xie \& Huson, 2015; default parameters; i.e., e-value $<1 \mathrm{e}-102$, hits per query $(-\mathrm{k})=$ 302 1), and (iii) Hotpep version 1 for short, conserved motifs in the PPR (Peptide Pattern

303 304 305 Recognition) library (Busk et al., 2017; default parameters; i.e., frequency $>2.6$, hits $>6$ ). For a detailed walk-through of the assembly, annotation, search for KEGG pathways, and comparative genomics analyses, please refer to the associated Jupyter notebook (Wilkins, 2020a).

\section{Identification of antibiotic resistance genes}

All three L. koalarum genome assemblies were uploaded to the Comprehensive Antibiotic Resistance Database (CARD version 3.0.7; Jia et al., 2017) and the ResFinder database version 5.1.0 (Zankari et al., 2012) to screen them for putative antibiotic resistance genes and their variants using blastn searches against CARD 2020 reference sequences using default parameters. The Resistance Gene Identifier (RGI) search pipeline was used to detect SNPs (single nucleotide polymorphisms) using the 'perfect, strict, complete genes only' criterion on their website. Briefly, antibiotic resistance genes were searched with nucleotide sequences as input. RGI first predicts complete open reading frames (ORFs) using Prodigal version 2.6.3. To find protein homologs in the CARD references, DIAMOND version 0.9.29 is used. The 'perfect' algorithm detects perfect matches of individual amino acids to positions in the curated reference sequences 
317 that had been previously associated with antibiotic resistance in other bacterial species (Alcock 318 et al., 2020).

319

\section{Results and discussion}

\section{Identification of isolates}

322 Besides the isolates identified as L. koalarum, we had several other colonies growing on the $323 \mathrm{BHI}+$ tannin plates, including isolates with $16 \mathrm{~S}$ rRNA gene sequences that matched Bacillus 324 cereus, Bacillus nealsonii, Bacillus sonorensis, and Escherichia coli. E. coli was the most 325 common species isolated.

326

327

Assembly taxonomy and gene annotation

328 The hybrid assembly generated was 2,608,483 bp in length with an N50 of 2,299,135 bp and a 329 coverage of 672. According to the marker gene analysis in CheckM, the assembly was $99.21 \%$ complete and less than 1\% contaminated with a GC content of 39.02\% (see Table 1 for additional details). One contig in the assembly appears to be a 3,899 bp long plasmid. This is indicated by circularity of that contig and positive matches to plasmids in related taxa when uploaded to the NCBI blast website for organism identification (Madden, 2003). The two most similar sequences on GenBank were a 71 percent similar sequence of Pasteurella multocida strain U-B411 plasmid pCCK411 (accession number FR798946.1) and a 70 percent similar sequence of Mannheimia haemolytica strain 48 plasmid pKKM48 (accession number MH316128.1). The putative plasmid sequence was deposited on FigShare (Wilkins \& Jospin, 338 2020).

The taxonomy of L. koalarum strain UCD-LQP1 was confirmed in three ways. First, a

341 phylogenetic tree was built based on the 16S rRNA gene extracted from the new assembly. This

342 16S rRNA gene sequence was aligned with other closely related 16S rRNA gene sequences on 343 the RDP website where 16S rRNA gene sequences of type strains are curated and sequences of 344 the closest relatives of a taxon are usually readily available (Dunitz et al., 2015). The 345 phylogenetically closest sequence to L. koalarum strain UCD-LQP1 in the 16S rRNA gene tree 346 was one from Lonepinella koalarum Y17189 (Fig. 1). Second, a whole genome concatenated

347 gene marker tree was inferred using the Genome Taxonomy Database (GTDB), as well as using 
348 PhyloSift, in parallel. In the GTDB tree, L. koalarum UCD-LQP1 was placed closest to 349 Actinobacillus succinogenes (GenBank accession number GCA_000017245.1). Note that as of 350 February 3, 2020, GTDB did not include any of the L. koalarum genome assemblies. In the 351 PhyloSift marker gene tree, all three L. koalarum assemblies clustered together, and $A$.

352 succinogenes was their phylogenetically closest neighbor (Fig. 2). Third, the average nucleotide 353 identity (ANI) between the genome of the L. koalarum type strain (DSM 10053; GenBank 354 accession number GCA_004339625.1) and the assembly of L. koalarum UCD-LQP1 was 355 estimated at 98.91 percent (standard deviation 0.17\%). The ANI value between L. koalarum 356 UCD-LQP1 and GCA_004565475.1 was 98.99 percent (SD 0.15\%) and the ANI value between 357 GCA_004339625.1 and GCA_004565475.1 was 99.99 percent (SD 0.08\%). Both of these 358 genome assemblies are based on the type strain of L. koalarum that originated in 1995 (Osawa et 359 al., 1995). All three approaches confirmed the taxonomy of strain UCD-LQP1 as Lonepinella

360

361

362 363 364 365 366

367 368 369 370 371 372 373 374 375

376 377 378 koalarum. Interestingly, A. succinogenes (GenBank accession number GCA_000017245.1) belongs now to a different taxonomic group based on GTDB taxonomy, namely Basfia succinogenes. Parks et al. (2018), among others (e.g., Hug et al., 2016; Castelle \& Banfield, 2018), have suggested relying on whole genome sequencing to reorganize the microbial tree of life, which will result in a majority of changes in classification and naming, and ultimately reflect a more accurate evolutionary relationship among groups (Parks et al., 2018).

There were no positive hits for any annotations associated with tannin degradation in the RAST SEED viewer. This negative result is in contrast to the experimentally verified tannin-degrading functions reported for this bacterium (Osawa et al., 1995). Moreover, tannic acid powder had been used to prepare the culturing medium and was expected to help select for bacterial tannin degraders. There are several potential explanations for the absence of any positive hits for tannins in the RAST database including (1) the genes responsible for tannin degradation in the assembly of L. koalarum UCD-LQP1 are not labeled as such, or (2) L. koalarum does not have any tannin-degradation functionality. We thus carried out additional sequence-based analyses searching for possible PCD degrading genes in the new assembly.

377 According to the annotation with PROKKA, there were 2,551 predicted genes and 2,479 protein coding genes. In comparison, eggNOG predicted 2,370 protein coding genes. Neither annotation 
379 included any genes annotated as "tannase". However, among the eggNOG predictions, there 380 were 79 genes putatively involved in Class 1.11 Xenobiotics biodegradation and the degradation 381 of plant secondary metabolites (Table 2). There are 20 KEGG pathways included in this group.

382 We searched for all twenty pathways in the assembly of L. koalarum strain UCD-LQP1 and 383 found positive hits in 13 pathways (Table 2). Each hit represents a translated amino acid 384 sequence from the assembly of $L$. koalarum UCD-LQP1 that is encoded by an individual gene in 385 a pathway. The largest proportion of hits $(\mathrm{n}=15)$ comprised putative enzymes that are members 386 in this KEGG class, but do not fall into a particular pathway (KEGG pathway ko00983: Drug 387 metabolism - other enzymes). Potential tannin-degrading genes might be found in this group but 388 have not been labeled as tannase genes because their sequences are not similar enough to any known tannase genes or because these tannase genes are not annotated in any database. The 390 second largest KEGG pathway was ko00362 benzoate degradation, followed by pathway ko00980 metabolism of xenobiotics by Cytochrome P450 and pathway ko00625 chloroalkane and chloroalkene degradation. KEGG pathways with fewer hits included the degradation 393 compounds such as aminobenzoate, xylene, naphthalene, dioxin, and chlorocyclohexane. Mapping individual genes onto KEGG pathways revealed continuous degradation chains for the following compounds: Azathioprine (pro-drug) to 6-Thioguanine (Supplementary Fig. S1); Aminobenzoate degradation; i.e., 4-Carboxy-2-hydroxymuconate semialdehyde to Pyruvate and 397 Oxaloacetate, which can then be fed into the citric acid cycle (Supplementary Fig. S2); 2398 Aminobenzene-sulfonate to Pyruvate, which, again, can be fed directly into Glycolysis or with another enzyme that was present (1.2.1.10) can be converted into Acetaldehyde, then Acetyl$400 \mathrm{CoA}$, and then fed into the Cytrate cycle (Supplementary Fig. S2). In the group of xenobiotics 401 metabolized by cytochrome P450 there were seven complete chains (Supplementary Fig. S3): 402 degradation of (i) benzo(a)pyrene, (ii) Aflatoxin B1, (iii) 1-Nitronaphtalene, (iv) 1,1-Dichloro403 ethylene, (v) Trichloroethylene, (vi) Bromobenzene, and (vii) 1,2-Dibromoethane. All of these 404 complete, putative conversion chains present in L. koalarum might explain further how this 405 member of the koala gut microbiome contributes to koala gastro-physiology (see discussion 406 below). Amino acid sequences encoded by putative PCD degrading genes in L. koalarum strain 407 UCD-LQP1 can be downloaded from FigShare (Wilkins, 2020b). A table linking eggNOG 408 annotations to positions in individual assemblies and translated amino acid sequences can be 
409 found in Supplementary Table S2. A complete table of all eggNOG annotations in the assembly 410 of L. koalarum strain UCD-LQP1 can be found in Supplementary Table S3.

411

412 Eucalyptus spp. leaves contain more than 100 different chemical compounds including 413 phenolics, terpenoids and lipids that are harmful for herbivores, even at low concentration 414 (Maghsoodlou et al., 2015). Koalas are highly specialized folivores feeding on these leaves. We 415 assumed that L. koalarum plays a beneficial role for koala hosts because some strains have 416 shown experimentally to be able to degrade tannins (Osawa, 1990; Osawa et al., 1995), and 417 tannic acid was used to isolate L. koalarum strain UCD-LQP1. Alas, we did not find any direct 418 evidence for tannase genes in the assembly of L. koalarum UCD-LQP1. However, genes 419 encoding several putative pathways involved in plant secondary metabolite degradation were 420 found in the assembly of L. koalarum UCD-LQP1. The predicted pathways included those for 421 degradation of compounds that had been extracted from Eucalyptus leaves (e.g., benzoate, 422 aminobenzoate, and chlorocyclohexane; Quinlivan et al., 2003; Marzoug et al., 2011; Sebei et 423 al., 2015; Maghsoodlou et al., 2015; Shiffman et al., 2017). Degradation of these PCDs might 424 explain the beneficial role that L. koalarum plays in the koala gut microbiome.

425

\section{Comparative genomics and unique genes in L. koalarum}

427 The GTDB tree clade used to extract related genomes of L. koalarum strain UCD-LQP1 428 consisted mostly of Haemophilus spp. $(\mathrm{n}=28)$, followed by Rodentibacter spp. $(\mathrm{n}=13)$, 429 Pasteurella spp. $(\mathrm{n}=5)$, Aggregibacter spp. $(\mathrm{n}=4)$, and seven other genera (Table S1). Whole 430 genome marker phylogenetic trees showed that not all genera were monophyletic. This can be 431 seen in Figure 2 in the way the coloring based on genus name does not group perfectly when taxa 432 are ordered according to their phylogenetic relationship. This was especially the case for 433 Haemophilus spp., which is shown in light purple. Some of the Haemophilus genomes were 434 grouped together, whereas others grouped with genomes labeled as Pasteurella spp., 435 Necropsobacter spp. and Avibacterium spp. One species of Rodentibacter (R. heylii) was closest 436 to Aggregatibacter spp. (yellow and pink in Fig. 2). Actinobacillus succinogenes and 437 Mannheimia succiniproducens grouped with Pasteurella spp., while the former was the most 438 closely related non-Lonepinella genome to L. koalarum strain UCD-LQP1. Here it is worth 439 noting that both A. succinogenes and M. succiniproducens have been renamed in the new GTDB 
440 taxonomy to Basfia succinogenes, most probably the most closely related taxon to L. koalarum 441 that has its genome sequenced to date. Twelve out of the 55 NCBI microbial genome assemblies 442 have different taxonomic names in the new GTDB taxonomy (Supplementary Table S1). For a 443 discussion of the re-organization and re-naming of the microbial tree of life based on whole 444 genome sequencing see above Assembly taxonomy and gene annotation. The whole genome 445 marker gene tree used to order genomes in Anvio's visualization can be downloaded from 446 FigShare (Wilkins, 2020c), as well as its corresponding amino acid alignment (Wilkins, 2020d). 447

448 Average nucleotide identities have been put forward as a measure of genomic relatedness among 449 bacteria that could help designate genera and be used besides the 16S rRNA gene as a taxonomic 450 marker (Barco et al., 2020). Moreover, it has been suggested to use an ANI threshold of larger 451 than 95\% to delineate bacterial species (Goris et al., 2007). Based on this definition, the genomes 452 used for the comparative genomic analysis with L. koalarum are all distinct species (heatmap in 453 Fig. 2). We created a second heatmap visualizing genomic relatedness at the $70 \%$ level 454 (Supplementary Fig. S4). This heatmap revealed several distinct clusters of closely related 455 456 genomes $v$ s. singleton genomes (i.e., taxa that did not group together with anything else at the 70 percent threshold): Cluster 1) Aggregatibacter spp., 2) first main Haemophilus spp. group, 3) Rodentibacter spp., 4) second main Haemophilus spp. group, 5) L. koalarum genome assemblies, and 6) two Necropsobacter spp. and another Haemophilus spp. Notably, Rodentibacter heylii, all Pasteurella spp., and Avibacterium paragallinarum did not cluster with anything. The heatmap is a way of visualizing sequence similarity groups and overall, it showed that the genera Haemophilus, Pasteurella and Rodentibacter do not represent coherent groups of species or genera. These three genera were found in several sub-groups (clusters in the ANI heatmap in Supplementary Fig. S4) that have been described previously based on a much larger sample size and a few marker genes (Naushad et al., 2015). Even some of the same singleton genomes were reported as their own branches in previous phylogenetic trees (Christensen et al., 2003). L. koalarum was placed in the middle of a group containing mostly Haemophilus, Pasteurella and Basfia species. Pasteurellaceae, the single constituent family of the order Pasteurellales hosts a diverse group of mostly pathogenic bacteria that had been assigned to this group based on phenotypic traits, often related to their pathology, and GC content (Mannheim, Pohl \& Holländer, 1980). For example, the genus Haemophilus includes a plethora of taxa that cause 
471 pneumonia and meningitis in humans, and Pasteurella have been associated with a range of 472 infectious diseases in cattle, fowl and pigs (Naushad et al., 2015). Moreover, since sequence473 based taxonomies have become more common, new genera have been created within each genus, 474 such as for example Aggregatibacter (Norskov-Lauritsen, 2006) or Avibacterium (Blackall et al., 475 2005). We believe that a work-over of the evolutionary genetic relationship of the Pasteurellales 476 is overdue.

477

478 The proportion of gene clusters that were unique to the three L. koalarum genome assemblies, 479 relative to 55 of their most closely related genomes, was large relative to the size of genes that 480 were unique to other genera in Anvio's pangenome analysis (Fig. 2). There were 282 gene 481 clusters that could exclusively be found in the three L. koalarum genome assemblies. Among 482 them, there were 136 gene clusters with complete sequences and COG annotation 483 (Supplementary Table S4). There were 36 gene clusters unique to L. koalarum strain UCD-LQP1 484 and 19 of these had complete sequences and COG annotations (Supplementary Table S5).

485

486 Out of the 136 gene clusters with known COG functions that were unique to the three $L$.

487 koalarum genome assemblies, 22 different gene clusters fell into the COG category 488 'Carbohydrate metabolism/transport'. This was the largest category, followed by 'Inorganic ion 489 transport' ( $\mathrm{n}=15)$, 'Cell wall', 'Transcription', and 'Energy production' ( $\mathrm{n}=11$, each), and 490 'Defense' ( $\mathrm{n}=7$; Table 3 and Supplementary Table S4). The translated amino acid sequences for 491 these gene clusters, extracted from L. koalarum strain UCD-LQP1, can be found in 492 Supplementary Table S6.

493

494 Gene clusters in the category 'Carbohydrate metabolism and transport' are discussed in detail 495 below. It is worth mentioning that several putative components of the phosphotransferase system 496 were unique to L. koalarum. This system transports sugars into bacteria including glucose, 497 mannose, fructose, and cellobiose. It can differ among bacterial species, mirroring the most 498 suitable carbon sources available in the environment where a species evolved (Tchieu et al., 499 2001). L. koalarum also stood out in terms of genes coding for cell wall components including 500 for example teichoic acid and other outer membrane proteins (Table 3 and Supplementary Table 501 S4). These outer membrane proteins are diverse and can significantly differ among bacterial 
502 species (Schleifer \& Kandler, 1972). A few other potentially unique gene clusters included genes 503 coding for type IV pilus assembly proteins for species-specific pili and fimbria (Proft \& Baker, 504 2009); defense mechanisms, such as putative bacteriophage resistance proteins, phage repressor 505 proteins; and drug transport and efflux pumps. Several of these factors are characteristic for 506 pathogenic bacteria (Craig, Pique \& Tainer, 2004). Here it is worth noting that a gram-negative 507 bacterium that was assigned to the genus Lonepinella based on 16S rRNA gene sequences 508 caused a human wound infection after a wildlife worker had been bitten by a koala (Sinclair et 509 al., 2019).

510

\section{Carbohydrate metabolism}

512 Since the majority of unique gene clusters in all three $L$. koalarum genome assemblies were 513 related to carbohydrate metabolism and transport, we decided to screen all three L. koalarum

514 assemblies for potential enzymes that assemble, modify, and breakdown oligo- and

515 polysaccharides. Using very stringent selection thresholds of the CAZy database where genes 516 coding for carbohydrate-active enzymes have to be identified by three different methods, we 517 found evidence for the presence of genes encoding 15 different glycoside hydrolase families, 518 three different carbohydrate esterase families, and nine different glycosyltransferase families 519 (Table 4). Note, gene families in L. koalarum are predicted to have these activities in 520 carbohydrate metabolism and transport based on characterized other members in the CAZy database, but we do not provide experimental evidence that $L$. koalarum performs these

522

523

524

525

526

527

528

529

530

531

532 activities. All 28 identified CAZy gene families had also been annotated in the 2,370 eggNOG annotations (Supplementary Table S3). Glycoside hydrolase families, GH2, GH31, GH32, GH43, and GH77 were only found in the three L. koalarum genome assemblies relative to the other taxa in the comparative genomic analysis (see also Table 3 and Supplementary Table S7). These five glycoside hydrolases are responsible for the hydrolysis of glycosidic bonds. Notably, when Lonepinella koalarum was isolated and described the first time as a phylogenetically and phenotypically novel group within the family Pasteurellaceae, enzyme activities were determined using commercially available oxidase/catalase tests as well as high-pressure liquid chromatography (Osawa et al., 1995). The new taxon in 1995 (first described L. koalarum) showed positive results for beta-galactosidase (putatively enzyme family $\mathrm{GH} 2$ ) and alphaamylase (putatively enzyme family GH77) and negative results for urease, arginine dihydrolase, 
533 lysine decarboxylase, and tryptophane desaminase in congruence with the sequence-based results 534 here.

535

536 Genes coding for oligosaccharide-degrading enzymes in the families GH1, GH2, GH3, GH42, 537 and GH43 have also been found in another study that was investigating koala and wombat

538

539

540

541

542

543

544

545

546

547

548

549

550

551

552

553

554

555

556

557

558

559

560

561

562

563

metagenomes (Shiffman et al., 2017). Especially GH2, GH3 and GH43 were relatively common in koala metagenomes, relative to wallaby foregut (Pope et al., 2010), cow rumen (Brulc et al., 2009), and termite hindgut (He et al., 2013) metagenomes, where these enzymes had also been characterized. These five glycoside hydrolase families comprise mostly oligosaccharidedegrading enzymes (Allgaier et al., 2010); i.e., they are able to break down a specific group of monosaccharide sugars in other bacteria that had been characterized for the CAZy database.

However, presumably the major components of koala diet that are difficult to digest for the host are plant secondary metabolites and plant cell walls in Eucalyptus leaves, and oligosaccharidedegrading enzymes only play a significant role in a koala's diet after other enzymes have already degraded cellulose in leaf plant cell walls (Moore et al., 2005). Oligosaccharides in Eucalyptus leaves will be absorbed by the koala in the small intestine and only a small fraction enter the caecum and colon. This means that the bacteria in the hindgut are most likely using their metabolic pathways to process the products of the degradation of complex carbohydrates with cross-feeding among microbiome members. The benefit of this activity to koala nutrition is not well understood. Interestingly, among the genes that code for the three carbohydrate-active enzyme families that were found exclusively in the assembly of $L$. koalarum strain UCD-LQP1, two were actual lignocellulases; i.e., microbial enzymes that hydrolyze the beta-1,4 linkages in cellulose (Allgaier et al., 2010): Enzyme family GH42 and CE4. GH42 enzymes have mostly been described in cellulose-degrading bacteria, archaea and fungi (Kosugi, Murashima \& Doi, 2002; Shipkowski \& Brenchley, 2006; Di Lauro et al., 2008). CE4 is a member of the carbohydrate esterase family, which groups enzymes that catalyze the de-acetylation of plant cell wall polysaccharides (Biely, 2012). Digestion of plant cell walls, (i.e., cellulose, hemicellulose, and lignin), could be a second explanation (besides PCD degradation) of how L. koalarum plays a beneficial role in the koala gut microbiome.

\section{Antibiotic resistance genes}

Peer) reviewing PDF | (2020:03:47130:2:0:NEW 11 Sep 2020) 
564 Screening the three L. koalarum genome assemblies against the ResFinder database did not result

565 in any detection of antibiotic resistance variants. However, there were three hits in the CARD

566 database. First, all three L. koalarum assemblies contained a gene coding for a translated amino

567 acid variant at a specific position (SNP R234F) that had been shown to confer resistance to

568 pulvomycin in other bacterial species based on CARD predictions. Secondly, a variant was

569 found to be encoded in all three L. koalarum genome assemblies that had been described before

570 in Haemophilus influenza mutant PBP3, conferring resistance to beta-lactam antibiotics

571 (cephalosporin, cephamycin, and penam) with SNPs D350N and S357N. The third result was an

572 amino acid position with reference to a protein homolog model in a Klebsiella pneumoniae

573 mutant, conferring resistance to the antibiotic efflux pump KpnH (including macrolide

574 antibiotics, fluoroquinolone, aminoglycoside, carbapenem, cephalosporin, penam, and penem).

575 These results are based on predictions from the CARD 2020 database. All three hits are

576 nucleotide sequences in the L. koalarum assemblies that are predicted to encode proteins that

577 showed the same amino acid variants as other bacterial species in the CARD database. We do

578 not know whether these variants confer antibiotic resistance in L. koalarum. Additional

579 experiments are necessary to confirm that these CARD predictions work for L. koalarum. The

580 corresponding nucleotide sequences and CARD output files are deposited on FigShare (UCD-

581 LQP1: Wilkins, 2020e; ATCC 700131: Wilkins, 2020f; and DSM 10053: Wilkins, 2020e).

582

583 Recommendations for future koala management strategies

584 In previous work, we identified L. koalarum as the most predictive taxon of koala survival 585 during antibiotic treatment and we suggested that this bacterium is important for koala health 586 (Dahlhausen et al., 2018). Here, we isolated a L. koalarum strain from the feces of a healthy

587 koala and sequenced and characterized its genome. We found several putative detoxification

588 pathways in L. koalarum strain UCD-LQP1 that could explain its potential beneficial role in the 589 koala gut for koala survival and fitness. Besides detoxification of plant secondary metabolites, 590 we found several putative genes involved in carbohydrate metabolism, particularly cellulose 591 degradation. Some of these genes were only found in L. koalarum assemblies and not in 55 of 592 their closely related genomes. Based on CARD predictions, the L. koalarum assemblies contain 593 some sequences that are similar to antibiotic resistance genes in other bacterial species. We

594 suggest confirming these antibiotic resistances in L. koalarum experimentally and testing the 
595 efficiency of these antibiotic compounds against Chlamydia infections in koalas. In light of the 596 various threats that koalas face, from chlamydia infection to wildfires (Polkinghorne, Hanger \& 597 Timms, 2013), and the growing interest in rescuing and treating them in sanctuaries and zoos, it 598 is important to identify beneficial members of their microbiome. This could (i) help decide 599 which antibiotic compounds to choose during chlamydia treatment in order to maximize 600 persistence of beneficial members in the koala gut microbiome, and (ii) guide the development 601 of probiotic cocktails during recovery (Jin Song et al., 2019).

602

603 Acknowledgements

604 We thank Céline Caseys ORCID: 0000-0003-4187-9018 for her idea to search eggNOG

605 annotations against KEGG Class 1.11 Xenobiotics biodegradation and metabolism pathways; 606 Chris Brown ORCID: 0000-0002-7758-6447 for his script to download assemblies in batch from 607 GenBank; Cassandra L. Ettinger ORCID: 0000-0001-7334-403X for valuable comments on the 608 pangenome analysis and help with revising the figures. We also thank Dr. Joseph Gillespie 609 ORCID: 0000-0002-5447-7264, Dr. Raphael Eisenhofer ORCID: 0000-0002-3843-0749 and two 610 anonymous reviewers for comments on the submitted manuscript. 


\section{References}

612 Adamczyk B, Simon J, Kitunen V, Adamczyk S, Smolander A. 2017. Tannins and their complex 613 interaction with different organic nitrogen compounds and enzymes: Old paradigms versus 614 recent advances. ChemistryOpen6:610-614. DOI: 10.1002/open.201700113.

615 Alcock BP, Raphenya AR, Lau TTY, Tsang KK, Bouchard M, Edalatmand A, Huynh W, 616 Nguyen A-LV, Cheng AA, Liu S, Min SY, Miroshnichenko A, Tran H-K, Werfalli RE, 617 Nasir JA, Oloni M, Speicher DJ, Florescu A, Singh B, Faltyn M, Hernandez-Koutoucheva 618 A, Sharma AN, Bordeleau E, Pawlowski AC, Zubyk HL, Dooley D, Griffiths E, Maguire F, 619 Winsor GL, Beiko RG, Brinkman FSL, Hsiao WWL, Domselaar GV, McArthur AG. 2020. 620 CARD 2020: Antibiotic resistome surveillance with the comprehensive antibiotic resistance 621 database. Nucleic Acids Research 48:D517-D525. DOI: 10.1093/nar/gkz935.

Alfano N, Courtiol A, Vielgrader H, Timms P, Roca AL, Greenwood AD. 2015. Variation in 623 koala microbiomes within and between individuals: Effect of body region and captivity status. Scientific Reports 5:10189. DOI: 10.1038/srep10189.

625

626

627

628

629

630

631

632

633

Allgaier M, Reddy A, Park JI, Ivanova N, D’haeseleer P, Lowry S, Sapra R, Hazen TC, Simmons BA, Vander Gheynst JS, Hugenholtz P. 2010. Targeted discovery of glycoside hydrolases from a switchgrass-adapted compost community. PLoS One 5:e8812. DOI: 10.1371/journal.pone.0008812.

Altschul SF, Gish W, Miller W, Myers EW, Lipman DJ. 1990. Basic local alignment search tool. Journal of Molecular Biology 215:403-410. DOI: 10.1016/S0022-2836(05)80360-2.

Aziz RK, Bartels D, Best AA, DeJongh M, Disz T, Edwards RA, Formsma K, Gerdes S, Glass EM, Kubal M, Meyer F, Olsen GJ, Olson R, Osterman AL, Overbeek RA, McNeil LK, Paarmann D, Paczian T, Parrello B, Pusch GD, Reich C, Stevens R, Vassieva O, Vonstein 
634

635

636

637

638

639

640

641

642

643

644

645

646

647

648

649

650

651

652

653

654

655

656

V, Wilke A, Zagnitko O. 2008. The RAST Server: Rapid annotations using subsystems technology. BMC Genomics 9:75. DOI: 10.1186/1471-2164-9-75.

Barco RA, Garrity GM, Scott JJ, Amend JP, Nealson KH, Emerson D. 2020. A genus definition for bacteria and archaea based on a standard genome relatedness index. mBio 11. DOI: 10.1128/mBio.02475-19.

Biely P. 2012. Microbial carbohydrate esterases deacetylating plant polysaccharides. Biotechnology Advances 30:1575-1588. DOI: 10.1016/j.biotechadv.2012.04.010.

Blackall PJ, Christensen H, Beckenham T, Blackall LL, Bisgaard M. 2005. Reclassification of Pasteurella gallinarum, [Haemophilus] paragallinarum, Pasteurella avium and Pasteurella volantium as Avibacterium gallinarum gen. nov., comb. nov., Avibacterium paragallinarum comb. nov., Avibacterium avium comb. nov. and Avibacterium volantium comb. nov. International Journal of Systematic and Evolutionary Microbiology 55:353-362.

Blyton MDJ, Soo RM, Whisson D, Marsh KJ, Pascoe J, Le Pla M, Foley M, Hugenholtz P, Moore BD. 2019. Faecal inoculations alter the gastrointestinal microbiome and allow dietary expansion in a wild specialist herbivore, the koala. Animal Microbiome 1:6. DOI: 10.1186/s42523-019-0008-0.

Brice KL, Trivedi P, Jeffries TC, Blyton MDJ, Mitchell C, Singh BK, Moore BD. 2019. The koala (Phascolarctos cinereus) fecal microbiome differs with diet in a wild population. PeerJ 7:e6534. DOI: 10.7717/peerj.6534.

Brulc JM, Antonopoulos DA, Miller MEB, Wilson MK, Yannarell AC, Dinsdale EA, Edwards RE, Frank ED, Emerson JB, Wacklin P, Coutinho PM, Henrissat B, Nelson KE, White BA. 2009. Gene-centric metagenomics of the fiber-adherent bovine rumen microbiome reveals forage specific glycoside hydrolases. Proceedings of the National Academy of Sciences of 
the United States of America 106:1948-1953. DOI: 10.1073/pnas.0806191105.

658 Buchfink B, Xie C, Huson DH. 2015. Fast and sensitive protein alignment using DIAMOND.

659 Nature Methods 12:59-60. DOI: 10.1038/nmeth.3176.

660 Bushnell B. 2014. BBMap: A fast, accurate, splice-aware aligner. Lawrence Berkeley National

661 Lab. (LBNL), Berkeley, CA (United States).

662 Busk PK, Pilgaard B, Lezyk MJ, Meyer AS, Lange L. 2017. Homology to peptide pattern for 663 annotation of carbohydrate-active enzymes and prediction of function. BMC Bioinformatics 664 18:214. DOI: $10.1186 / \mathrm{s} 12859-017-1625-9$.

665 Callaghan J, McAlpine C, Mitchell D, Thompson J, Bowen M, Rhodes J, de Jong C,

666 Domalewski R, Scott A. 2011. Ranking and mapping koala habitat quality for conservation 667 planning on the basis of indirect evidence of tree-species use: a case study of Noosa Shire, 668 south-eastern Queensland. Wildlife Research 38:89. DOI: 10.1071/wr07177.

669 Cantarel BL, Coutinho PM, Rancurel C, Bernard T, Lombard V, Henrissat B. 2009. The 670 Carbohydrate-Active EnZymes database (CAZy): An expert resource for glycogenomics. $671 \quad$ Nucleic Acids Research 37:D233-8. DOI: 10.1093/nar/gkn663.

672 Castelle CJ, Banfield JF. 2018. Major new microbial groups expand diversity and alter our 673 understanding of the tree of life. Cell 172:1181-1197. DOI: 10.1016/j.cell.2018.02.016.

674 Chaumeil PA, Hugenholtz P, Parks DH. 2018. GTDB-Tk: A toolkit to classify genomes with the 675 Genome Taxonomy Database. Bioinformatics 36:1925-1927. DOI:

$676 \quad$ 10.1093/bioinformatics/btz848.

677 Christensen H, Bisgaard M, Bojesen AM, Mutters R, Olsen JE. 2003. Genetic relationships 678 among avian isolates classified as Pasteurella haemolytica, “Actinobacillus salpingitidis” or 679 Pasteurella anatis with proposal of Gallibacterium anatis gen. nov., comb. nov. and 
description of additional genomospecies within Gallibacterium gen. nov. International Journal of Systematic and Evolutionary Microbiology 53:275-287.

682 Cole JR, Wang Q, Fish JA, Chai B, McGarrell DM, Sun Y, Brown CT, Porras-Alfaro A, Kuske 683 CR, Tiedje JM. 2014. Ribosomal Database Project: Data and tools for high throughput 684 rRNA analysis. Nucleic Acids Research 42:D633-42. DOI: 10.1093/nar/gkt1244.

685 Craig L, Pique ME, Tainer JA. 2004. Type IV pilus structure and bacterial pathogenicity. Nature 686 Reviews in Microbiology 2:363-378. DOI: 10.1038/nrmicro885.

687 Dahlhausen KE, Doroud L, Firl AJ, Polkinghorne A, Eisen JA. 2018. Characterization of shifts 688 of koala (Phascolarctos cinereus) intestinal microbial communities associated with $689 \quad$ antibiotic treatment. PeerJ 6:e4452. DOI: $10.7717 /$ peerj.4452.

690 Darling AE, Jospin G, Lowe E, Matsen FA 4th, Bik HM, Eisen JA. 2014. PhyloSift:

691 Phylogenetic analysis of genomes and metagenomes. PeerJ 2:e243. DOI:

$692 \quad 10.7717 /$ peerj.243.

693 Delmont TO, Eren AM. 2018. Linking pangenomes and metagenomes: the Prochlorococcus 694 metapangenome. PeerJ 6:e4320. DOI: 10.7717/peerj.4320.

695 Desclozeaux M, Robbins A, Jelocnik M, Khan SA, Hanger J, Gerdts V, Potter A, Polkinghorne 696 A, Timms P. 2017. Immunization of a wild koala population with a recombinant Chlamydia 697 pecorum Major Outer Membrane Protein (MOMP) or Polymorphic Membrane Protein 698 (PMP) based vaccine: New insights into immune response, protection and clearance. PLoS 699 One 12:e178786. DOI: 10.1371/journal.pone.0178786.

700 Di Lauro B, Strazzulli A, Perugino G, La Cara F, Bedini E, Corsaro MM, Rossi M, Moracci M. 7012008 . Isolation and characterization of a new family $42 \beta$-galactosidase from the 702 thermoacidophilic bacterium Alicyclobacillus acidocaldarius: Identification of the active 
703

704

705

706

707

708

709

710

711

712

713

714

715

716

717

718

719

720

721

722

723

724 725

site residues. Biochimica et Biophysica Acta (BBA) - Proteins and Proteomics 1784:292301. DOI: 10.1016/j.bbapap.2007.10.013.

van Dongen S, Abreu-Goodger C. 2012. Using MCL to extract clusters from networks. Methods in Molecular Biology 804:281-295. DOI: 10.1007/978-1-61779-361-5_15.

Dunitz MI, Lang JM, Jospin G, Darling AE, Eisen JA, Coil DA. 2015. Swabs to genomes: A comprehensive workflow. PeerJ 3:e960. DOI: 10.7717/peerj.960.

Eddy SR. 1998. Profile hidden Markov models. Bioinformatics 14:755-763. DOI: 10.1093/bioinformatics/14.9.755.

Edgar RC. 2004. MUSCLE: A multiple sequence alignment method with reduced time and space complexity. BMC Bioinformatics 5:113. DOI: 10.1186/1471-2105-5-113.

Eren AM, Esen ÖC, Quince C, Vineis JH, Morrison HG, Sogin ML, Delmont TO. 2015. Anvi'o: An advanced analysis and visualization platform for 'omics data. PeerJ 3:e1319. DOI: $10.7717 /$ peerj.1319.

Foley WJ, Lassak EV, Brophy J. 1987. Digestion and absorption of Eucalyptus essential oils in greater glider (Petauroide svolans) and brushtail possum (Trichosurus vulpecula). Journal of Chemical Ecology 13:2115-2130. DOI: 10.1007/BF01012875.

Freeland WJ, Janzen DH. 1974. Strategies in herbivory by mammals: The role of plant secondary compounds. The American Naturalist 108:269-289. DOI: 10.1086/282907.

Gleadow RM, Haburjak J, Dunn JE, Conn ME, Conn EE. 2008. Frequency and distribution of cyanogenic glycosides in Eucalyptus L'Hérit. Phytochemistry 69:1870-1874. DOI: 10.1016/j.phytochem.2008.03.018.

Goel G, Puniya AK, Aguilar CN, Singh K. 2005. Interaction of gut microflora with tannins in feeds. Die Naturwissenschaften 92:497-503. DOI: 10.1007/s00114-005-0040-7. 
726 Goris J, Konstantinidis KT, Klappenbach JA, Coenye T, Vandamme P, Tiedje JM. 2007. DNA-

727 DNA hybridization values and their relationship to whole-genome sequence similarities.

728 International Journal of Systematic and Evolutionary Microbiology 57:81-91.

729 Gurevich A, Saveliev V, Vyahhi N, Tesler G. 2013. QUAST: Quality assessment tool for 730 genome assemblies. Bioinformatics 29:1072-1075. DOI: 10.1093/bioinformatics/btt086.

731 Hammer TJ, Bowers MD. 2015. Gut microbes may facilitate insect herbivory of chemically 732 defended plants. Oecologia 179:1-14. DOI: 10.1007/s00442-015-3327-1.

733 He S, Ivanova N, Kirton E, Allgaier M, Bergin C, Scheffrahn RH, Kyrpides NC, Warnecke F, 734 Tringe SG, Hugenholtz P. 2013. Comparative metagenomic and metatranscriptomic 735 analysis of hindgut paunch microbiota in wood- and dung-feeding higher termites. PLoS $736 \quad$ One 8:e61126. DOI: 10.1371/journal.pone.0061126.

737 Higgins AL, Bercovitch FB, Tobey JR, Andrus CH. 2011. Dietary specialization and Eucalyptus 738 species preferences in Queensland koalas (Phascolarctos cinereus). Zoo Biology 30:52-58. 739 DOI: $10.1002 /$ zoo.20312.

740 Hug LA, Baker BJ, Anantharaman K, Brown CT, Probst AJ, Castelle CJ, Butterfield CN, 741 Hernsdorf AW, Amano Y, Ise K, Suzuki Y, Dudek N, Relman DA, Finstad KM, Amundson 742 R, Thomas BC, Banfield JF. 2016. A new view of the tree of life. Nature Microbiology $743 \quad$ 1:16048. DOI: 10.1038/nmicrobiol.2016.48.

744 Hyatt D, Chen G-L, Locascio PF, Land ML, Larimer FW, Hauser LJ. 2010. Prodigal:

745 Prokaryotic gene recognition and translation initiation site identification. BMC 746 Bioinformatics 11:119. DOI: 10.1186/1471-2105-11-119.

747 Jensen LJ, Julien P, Kuhn M, von Mering C, Muller J, Doerks T, Bork P. 2008. eggNOG:

748 Automated construction and annotation of orthologous groups of genes. Nucleic Acids 
Research 36:D250-4. DOI: 10.1093/nar/gkm796.

750 Jia B, Raphenya AR, Alcock B, Waglechner N, Guo P, Tsang KK, Lago BA, Dave BM, Pereira

751 S, Sharma AN, Doshi S, Courtot M, Lo R, Williams LE, Frye JG, Elsayegh T, Sardar D,

752 Westman EL, Pawlowski AC, Johnson TA, Brinkman FSL, Wright GD, McArthur AG.

753 2017. CARD 2017: Expansion and model-centric curation of the comprehensive antibiotic

754 resistance database. Nucleic Acids Research 45:D566-D573. DOI: 10.1093/nar/gkw1004.

755 Jin Song S, Woodhams DC, Martino C, Allaband C, Mu A, Javorschi-Miller-Montgomery S,

756 Suchodolski JS, Knight R. 2019. Engineering the microbiome for animal health and

757 conservation. Experimental Biology and Medicine 244:494-504. DOI:

$758 \quad 10.1177 / 1535370219830075$.

759 Johnson RN, O’Meally D, Chen Z, Etherington GJ, Ho SYW, Nash WJ, Grueber CE, Cheng Y,

760 Whittington CM, Dennison S, Peel E, Haerty W, O’Neill RJ, Colgan D, Russell TL,

761 Alquezar-Planas DE, Attenbrow V, Bragg JG, Brandies PA, Chong AY-Y, Deakin JE, Di

762 Palma F, Duda Z, Eldridge MDB, Ewart KM, Hogg CJ, Frankham GJ, Georges A, Gillett

763 AK, Govendir M, Greenwood AD, Hayakawa T, Helgen KM, Hobbs M, Holleley CE,

764 Heider TN, Jones EA, King A, Madden D, Graves JAM, Morris KM, Neaves LE, Patel HR,

765 Polkinghorne A, Renfree MB, Robin C, Salinas R, Tsangaras K, Waters PD, Waters SA,

766 Wright B, Wilkins MR, Timms P, Belov K. 2018. Adaptation and conservation insights

767 from the koala genome. Nature Genetics 50:1102-1111. DOI: 10.1038/s41588-018-0153-5.

768 Jospin G. 2018. PhyloSift markers database. Figshare. DOI:

769 https://doi.org/10.6084/m9.figshare.5755404.v4

770 Kanehisa M, Goto S. 2000. KEGG: Kyoto encyclopedia of genes and genomes. Nucleic Acids

$771 \quad$ Research 28:27-30. DOI: 10.1093/nar/28.1.27. 
772 Kohl KD, Weiss RB, Cox J, Dale C, Dearing MD. 2014. Gut microbes of mammalian herbivores 773 facilitate intake of plant toxins. Ecology Letters 17:1238-1246. DOI: 10.1111/ele.12329.

774 Kohl KD, Denise Dearing M. 2016. The woodrat gut microbiota as an experimental system for 775 understanding microbial metabolism of dietary toxins. Frontiers in Microbiology 7. DOI: $776 \quad 10.3389 /$ fmicb.2016.01165.

777 Kohl KD, Stengel A, Denise Dearing M. 2016. Inoculation of tannin-degrading bacteria into 778 novel hosts increases performance on tannin-rich diets. Environmental Microbiology 779 18:1720-1729. DOI: 10.1111/1462-2920.12841.

780 Koren S, Walenz BP, Berlin K, Miller JR, Bergman NH, Phillippy AM. 2017. Canu: Scalable 781 and accurate long-read assembly via adaptive k-mer weighting and repeat separation. 782 Genome Research 27:722-736. DOI: 10.1101/gr.215087.116.

783 Kosugi A, Murashima K, Doi RH. 2002. Characterization of two noncellulosomal subunits, 784 ArfA and BgaA, from Clostridium cellulovorans that cooperate with the cellulosome in 785 plant cell wall degradation. Journal of Bacteriology 184:6859-6865. DOI:

$786 \quad 10.1128 / \mathrm{jb} .184 .24 .6859-6865.2002$.

787 Lawler IR, Foley WJ, Eschler BM. 2000. Foliar concentration of a single toxin creates habitat 788 patchiness for a marsupial folivore. Ecology 81:1327. DOI: $10.2307 / 177211$.

789 Letunic I, Bork P. 2019. Interactive Tree Of Life (iTOL) v4: Recent updates and new 790 developments. Nucleic Acids Research 47:W256-W259. DOI: 10.1093/nar/gkz239.

791 Liu B, dos Santos BM, Kanagendran A, Neilson E, Niinemets Ü. 2019. Ozone and wounding 792 stresses differently alter the temporal variation in formylated phloroglucinols in Eucalyptus 793 globulus leave. Metabolites 9:46. DOI: 10.3390/metabo9030046.

794 Lombard V, Golaconda Ramulu H, Drula E, Coutinho PM, Henrissat B. 2014. The carbohydrate- 

active enzymes database (CAZy) in 2013. Nucleic Acids Research 42:D490-5. DOI: 10.1093/nar/gkt1178.

797 Looft T, Levine UY, Stanton TB. 2013. Cloacibacillus porcorum sp. nov., a mucin-degrading 798 bacterium from the swine intestinal tract and emended description of the genus 799 Cloacibacillus. International Journal of Systematic and Evolutionary Microbiology

Madden T. 2003. The BLAST Sequence Analysis Tool. National Center for Biotechnology

803

804 805 806 807 808 809 810 811 812 813 814 815 816 817 Information (US).

Maghsoodlou MT, Kazemipoor N, Valizadeh J, Falak Nezhad Seifi M, Rahneshan N. 2015. Essential oil composition of Eucalyptus microtheca and Eucalyptus viminalis. Avicenna Journal of Phytomedicine 5:540-552.

Mannheim W, Pohl S, Holländer R. 1980. On the taxonomy of Actinobacillus, Haemophilus, and Pasteurella: DNA base composition, respiratory quinones, and biochemical reactions of representative collection cultures (author's transl). Zentralblatt fur Bakteriologie. 1. Abt. Originale. A: Medizinische Mikrobiologie, Infektionskrankheiten und Parasitologie 246:512-540.

Marsh KJ, Foley WJ, Cowling A, Wallis IR. 2003. Differential susceptibility to Eucalyptus secondary compounds explains feeding by the common ringtail (Pseudocheirus peregrinus) and common brushtail possum (Trichosurus vulpecula). Journal of Comparative Physiology. B, Biochemical, Systemic, and Environmental Physiology 173:69-78. DOI: $10.1007 / \mathrm{s} 00360-002-0318-4$.

Marzoug HNB, Romdhane M, Lebrihi A, Mathieu F, Couderc F, Abderraba M, Khouja ML, Bouajila J. 2011. Eucalyptus oleosa essential oils: Chemical composition and antimicrobial 
818

819

820

821

822

823

824

825

826

827

828

829

830

831

832

833

834

835

836

837

838

839

840

and antioxidant activities of the oils from different plant parts (stems, leaves, flowers and fruits). Molecules 16:1695-1709. DOI: 10.3390/molecules16021695.

Miller MA, Pfeiffer W, Schwartz T. 2010. Creating the CIPRES Science Gateway for inference of large phylogenetic trees. In: 2010 Gateway Computing Environments Workshop (GCE). 1-8. DOI: 10.1109/GCE.2010.5676129.

Moore BD, Foley WJ. 2005. Tree use by koalas in a chemically complex landscape. Nature 435:488-490. DOI: 10.1038/nature03551.

Moore BD, Foley WJ, Wallis IR, Cowling A, Handasyde KA. 2005. Eucalyptus foliar chemistry explains selective feeding by koalas. Biology Letters 1:64-67. DOI:

10.1098/rsbl.2004.0255.

Naushad S, Adeolu M, Goel N, Khadka B, Al-Dahwi A, Gupta RS. 2015. Phylogenomic and molecular demarcation of the core members of the polyphyletic Pasteurellaceae genera Actinobacillus, Haemophilus, and Pasteurella. International Journal of Genomics and Proteomics 2015. DOI: 10.1155/2015/198560.

Ngo S, Kong S, Kirlich A, McKinnon RA, Stupans I. 2000. Cytochrome P450 4A, peroxisomal enzymes and nicotinamide cofactors in koala liver. Comparative Biochemistry and Physiology Part C: Pharmacology, Toxicology and Endocrinology 127:327-334. DOI: 10.1016/s0742-8413(00)00160-2.

Norskov-Lauritsen N. 2006. Reclassification of Actinobacillus actinomycetemcomitans, Haemophilus aphrophilus, Haemophilus paraphrophilus and Haemophilus segnis as Aggregatibacter actinomycetemcomitans gen. nov., comb. nov., Aggregatibacter aphrophilus comb. nov. and Aggregatibacter segnis comb. nov., and emended description of Aggregatibacter aphrophilus to include V factor-dependent and V factor-independent 
841 isolates. International Journal of Systematic and Evolutionary Microbiology 56:2135-2146.

842 DOI: $10.1099 /$ ijs.0.64207-0.

843 Nyari S, Khan SA, Rawlinson G, Waugh CA, Potter A, Gerdts V, Timms P. 2018. Vaccination

844 of koalas (Phascolarctos cinereus) against Chlamydia pecorum using synthetic peptides

845 derived from the major outer membrane protein. PLoS One 13:e0200112. DOI:

$846 \quad$ 10.1371/journal.pone.0200112.

847 Osawa R. 1990. Formation of a clear zone on tannin-treated brain heart infusion agar by a

848 Streptococcus sp. isolated from feces of koalas. Applied and Environmental Microbiology

$849 \quad 56: 829-831$.

850 Osawa R. 1992. Tannin-protein complex-degrading Enterobacteria isolated from the alimentary

851 tract of koalas and a selective medium for their enumeration. Applied and Environmental

$852 \quad$ Microbiology 58:1754-1759.

853 Osawa R, Bird PS, Harbrow DJ, Ogimoto K, Seymour GJ. 1993. Microbiological studies of the

854 intestinal microflora of the koala, Phascolarctos cinereus: Colonization of the cecal wall by

855 tannin-protein-complex-degrading Enterobacteria. Australian Journal of Zoology 41:599.

$856 \quad$ DOI: $10.1071 /$ zo9930599.

857 Osawa R, Rainey F, Fujisawa T, Lang E, Busse HJ, Walsh TP, Stackebrandt E. 1995.

858 Lonepinella koalarum gen. nov., sp. nov., a new tannin-protein complex degrading

859 bacterium. Systematic and Applied Microbiology 18:368-373. DOI: 10.1016/s0723-

$860 \quad 2020(11) 80430-3$.

861 Overbeek R, Olson R, Pusch GD, Olsen GJ, Davis JJ, Disz T, Edwards RA, Gerdes S, Parrello

862 B, Shukla M, Vonstein V, Wattam AR, Xia F, Stevens R. 2014. The SEED and the Rapid 863 Annotation of microbial genomes using Subsystems Technology (RAST). Nucleic Acids 
865 Parks DH, Chuvochina M, Waite DW, Rinke C, Skarshewski A, Chaumeil P-A, Hugenholtz P. 866 2018. A standardized bacterial taxonomy based on genome phylogeny substantially revises 867 the tree of life. Nature Biotechnology 36:996-1004. DOI: 10.1038/nbt.4229.

868 Parks DH, Imelfort M, Skennerton CT, Hugenholtz P, Tyson GW. 2015. CheckM: Assessing the 869 quality of microbial genomes recovered from isolates, single cells, and metagenomes.

$870 \quad$ Genome Research 25:1043-1055. DOI: 10.1101/gr.186072.114.

871 Polkinghorne A, Hanger J, Timms P. 2013. Recent advances in understanding the biology, 872 epidemiology and control of chlamydial infections in koalas. Veterinary Microbiology 873 165:214-223. DOI: 10.1016/j.vetmic.2013.02.026.

874 Pope PB, Denman SE, Jones M, Tringe SG, Barry K, Malfatti SA, McHardy AC, Cheng J-. F, 875 Hugenholtz P, McSweeney CS, Morrison M. 2010. Adaptation to herbivory by the Tammar 876 wallaby includes bacterial and glycoside hydrolase profiles different from other herbivores. 877 Proceedings of the National Academy of Sciences 107:14793-14798. DOI: 10.1073/pnas. 1005297107.

879 Price MN, Dehal PS, Arkin AP. 2009. FastTree: Computing large minimum evolution trees with 880 profiles instead of a distance matrix. Molecular Biology and Evolution 26:1641-1650. DOI: $881 \quad 10.1093 / \mathrm{molbev} / \mathrm{msp} 077$.

882 Pritchard L, Glover RH, Humphris S, Elphinstone JG, Toth IK. 2016. Genomics and taxonomy 883 in diagnostics for food security: soft-rotting enterobacterial plant pathogens. Analytical 884 Methods 8:12-24. DOI: 10.1039/C5AY02550H.

885 Proft T, Baker EN. 2009. Pili in Gram-negative and Gram-positive bacteria — structure, 886 assembly and their role in disease. Cellular and Molecular Life Sciences 66:613-635. DOI: 
887

888

889

890

891

892

893

894

895

896

897

898

899

900

901

902

903

904

905

906

907

908

909

\section{$10.1007 / \mathrm{s} 00018-008-8477-4$.}

Quinlivan EP, Roje S, Basset G, Shachar-Hill Y, Gregory JF 3rd, Hanson AD. 2003. The folate precursor p-aminobenzoate is reversibly converted to its glucose ester in the plant cytosol. The Journal of Biological Chemistry 278:20731-20737. DOI: 10.1074/jbc.M302894200.

R Development Core Team. 2013. A language and environment for statistical computing. $R$ Foundation for Statistical Computing, Vienna, Austria.

Schleifer KH, Kandler O. 1972. Peptidoglycan types of bacterial cell walls and their taxonomic implications. Bacteriological Reviews 36:407.

Sebei K, Sakouhi F, Herchi W, Khouja ML, Boukhchina S. 2015. Chemical composition and antibacterial activities of seven Eucalyptus species essential oils leaves. Biological Research 48:7. DOI: 10.1186/0717-6287-48-7.

Seemann T. 2014. Prokka: Rapid prokaryotic genome annotation. Bioinformatics 30:2068-2069. DOI: 10.1093/bioinformatics/btu153.

Shiffman ME, Soo RM, Dennis PG, Morrison M, Tyson GW, Hugenholtz P. 2017. Gene and genome-centric analyses of koala and wombat fecal microbiomes point to metabolic specialization for digestion. PeerJ 5:e4075. DOI: 10.7717/peerj.4075.

Shipkowski S, Brenchley JE. 2006. Bioinformatic, genetic, and biochemical evidence that some glycoside hydrolase family 42 beta-galactosidases are arabinogalactan type I oligomer hydrolases. Applied and Environmental Microbiology 72:7730-7738. DOI: 10.1128/AEM.01306-06.

Sinclair HA, Chapman P, Omaleki L, Bergh H, Turni C, Blackall P, Papacostas L, Braslins P, Sowden D, Nimmo GR. 2019. Identification of Lonepinella sp. in koala bite wound infections, Queensland, Australia. Emerging Infectious Diseases 25:153-156. DOI: 
910

911

912

913

914

915

916

917

918

919

920

921

922

923

924

925

926

927

928

929

930

931

932

10.3201/eid2501.171359.

SNPsaurus | GENOMES to GENOTYPES. Available at https://www.snpsaurus.com (accessed August 20, 2019).

Stackebrandt E, Goodfellow M. 1991. Nucleic Acid Techniques in Bacterial Systematics. John Wiley \& Son Ltd.

Stucky BJ. 2012. SeqTrace: A graphical tool for rapidly processing DNA sequencing chromatograms. Journal of Biomolecular Techniques: JBT 23:90-93. DOI: 10.7171/jbt.122303-004.

Tchieu JH, Norris V, Edwards JS, Saier MH Jr. 2001. The complete phosphotransferase system in Escherichia coli. Journal of Molecular Microbiology and Biotechnology 3:329-346.

Turner S, Pryer KM, Miao VP, Palmer JD. 1999. Investigating deep phylogenetic relationships among cyanobacteria and plastids by small subunit rRNA sequence analysis. The Journal of Eukaryotic Microbiology 46:327-338. DOI: 10.1111/j.1550-7408.1999.tb04612.x.

Waterman PG, Mbi CN, McKey DB, Stephen Gartlan J. 1980. African rainforest vegetation and rumen microbes: Phenolic compounds and nutrients as correlates of digestibility. Oecologia 47:22-33. DOI: 10.1007/bf00541771.

Waugh C, Khan SA, Carver S, Hanger J, Loader J, Polkinghorne A, Beagley K, Timms P. 2016. A prototype recombinant-protein based Chlamydia pecorum vaccine results in reduced Chlamydial burden and less clinical disease in free-ranging koalas (Phascolarctos cinereus). PLoS One 11:e0146934. DOI: 10.1371/journal.pone.0146934.

Wick RR, Judd LM, Gorrie CL, Holt KE. 2017. Unicycler: Resolving bacterial genome assemblies from short and long sequencing reads. PLoS Computational Biology 8:e1005595. DOI: 10.1371/journal.pcbi.1005595. 
933 Wilkins LGE. 2020a. Notebook Lonepinella koalarum comparative genomic analysis. Figshare.

934 DOI: https://doi.org/10.6084/m9.figshare.11678262.v1

935 Wilkins LGE. 2020b. Putative amino acid sequences involved in xenobiotics biodegradation and 936 metabolism. Figshare. DOI: https://doi.org/10.6084/m9.figshare.11719278.v1

937 Wilkins LGE. 2020c. Tree file RAxML to order genomes in Anvi'o. Figshare. DOI:

938 https://doi.org/10.6084/m9.figshare.11719338.v1

939 Wilkins LGE. 2020d. Amino acid alignment from PhyloSift to order genomes in Anvi'o.

$940 \quad$ Figshare. DOI: https://doi.org/10.6084/m9.figshare.11719326.v1

941 Wilkins LGE. 2020e. Antibiotic resistance genes in GCA_004339625; Lonepinella koalarum.

942 Figshare. DOI: https://doi.org/10.6084/m9.figshare.11760249.v1

943 Wilkins LGE. 2020f. Antibiotic resistance genes in GCA_004565475; Lonepinella koalarum.

944 Figshare. DOI: https://doi.org/10.6084/m9.figshare.11760219.v2

945 Wilkins LGE. 2020g. Anvi'o genomes database for Lonepinella koalarum. Figshare. DOI:

946 https://doi.org/10.6084/m9.figshare.11760285.v1

947 Wilkins LGE. 2020h. Anvi'o profile for Lonepinella koalarum. Figshare. DOI:

948 https://doi.org/10.6084/m9.figshare.11760324.v1

949 Wilkins LGE, Coil DA. 2020a. 16S rRNA gene based alignment. Figshare. DOI:

$950 \quad$ https://doi.org/10.6084/m9.figshare.11678265.v1

951 Wilkins LGE, Coil DA. 2020b. 16S rRNA gene phylogenetic tree. Figshare. DOI:

952 https://doi.org/10.6084/m9.figshare.11678259.v1

953 Wilkins LGE, Ettinger CL, Jospin G, Eisen JA. 2019. Metagenome-assembled genomes provide 954 new insight into the microbial diversity of two thermal pools in Kamchatka, Russia.

955 Scientific Reports 9:3059. DOI: 10.1038/s41598-019-39576-6. 
956 Wilkins LGE, Jospin G. 2020. Contig 9 - potential Lonepinella koalarum plasmid. Figshare.

957 DOI: https://doi.org/10.6084/m9.figshare.11717895.v1

958 Yin Y, Mao X, Yang J, Chen X, Mao F, Xu Y. 2012. dbCAN: A web resource for automated 959 carbohydrate-active enzyme annotation. Nucleic Acids Research 40:W445-51. DOI:

$960 \quad 10.1093 /$ nar/gks479.

961 Zankari E, Hasman H, Cosentino S, Vestergaard M, Rasmussen S, Lund O, Aarestrup FM, 962 Larsen MV. 2012. Identification of acquired antimicrobial resistance genes. Journal of 963 Antimicrobial Chemotherapy 67:2640-2644. DOI: 10.1093/jac/dks261. 


\section{Figure 1}

16S rRNA gene phylogenetic placement of Lonepinella koalarum strain UCD-LQP1

The $16 \mathrm{~S}$ rRNA gene was extracted from the $L$. koalarum genome assembly by searching for 'ssu rRNA' in the RAST function search of the SEED genome browser (Aziz et al., 2008; Overbeek et al., 2014). Included are all known 16S rRNA sequences in the Pasteurellaceae family and one outgroup, Agarivoran spp. Nodes and tip labels are colored corresponding to the Anvi'o profile in Figure 2; i.e., red: Lonepinella koalarum (Unicycler: assembly of $L$. koalarum strain UCD-LQP1, in bold and marked with a star), dark purple: Pasteurella spp., light purple: Haemophilus spp., orange: Actinobacillus spp., pink: Aggregatibacter spp., and green: Mannheimia spp. Black are genera that were not used in Figure 2, and brown depicts the outgroup Agarivoran spp. 
Tree scale: 0.01 H.

GQ200591 Agarivorans gilvus

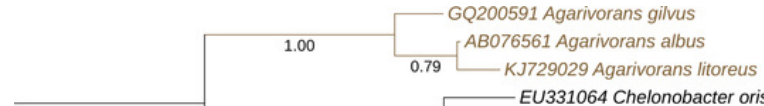

EU331064 Chelonobacter oris

$0.94 \quad$ AY362926 Pasteurella testudinis

AF024525 Actinobacillus succinogenes

M75058 Avibacterium avium

$1.001 .00{ }^{-1}$ AY498868 Avibacterium paragallinarum

0.41 AY362921 Avibacterium gallinarum

0.02M75070 Avibacterium volantium

0.81 Y09653 Actinobacillus scotiae

0.97 $1.00 \_$AY362889 Actinobacillus delphinicola

$0\left[15 \quad 1.00-\begin{array}{c}\text { AY216868 Volucribacter psittacicida } \\ \text { AY216869 Volucribacter amazonae }\end{array}\right.$

$0.97 \quad$ LO6078 Gallibacterium salpingitidis

$0.98 \longrightarrow$ EU339199 Gallibacterium trehalosifermentans

0.83 AF228001 Gallibacterium anatis

U65583 Actinobas5 Gallibacterium melopsittaci

- JF506642 Haemophilus sputorum

$\square_{0.70} 9.901 .00 \quad$ AJ290755 Haemophilus pittmaniae

$0.75 \quad 0.06 \quad$ Ar 362908 Haemophilus parainfluenzae

U66491 Pasteurella aerogenes

$0.96 \square$ AY362923 Pasteurella mairii

$93 \quad$ KF875567 Mesocricetibacter intestinalis

$0.76 \begin{aligned} & \text { KF875567 Mesocricetibacter intestinalis } \\ & 0.83\end{aligned}$

1.00

AY508816 Nicoletella semolina

d.94 AF294412 Pasteurella multocida

1.00 - AF294411 Pasteurella multocida

0.79F294410 Pasteurella multocida

1.00 AY362919 Pasteurella canis

0.57 - AY362920 Pasteurella dagmatis

0.79 YY362925 Pasteurella stomatis

0.98_ M75052 Pasteurella oralis

$0.87 \quad$ AY362905 Haemophilus aegyptius

1.00 M35019 Haemophilus influenzae

$0.99 \quad$ JN175335 Haemophilus haemolyticus

$0.93 \square$ M75043 Aggregatibacter segnis

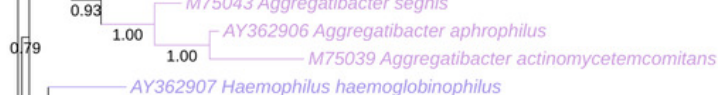

0.97 AY362909 Haemophilus parasuis

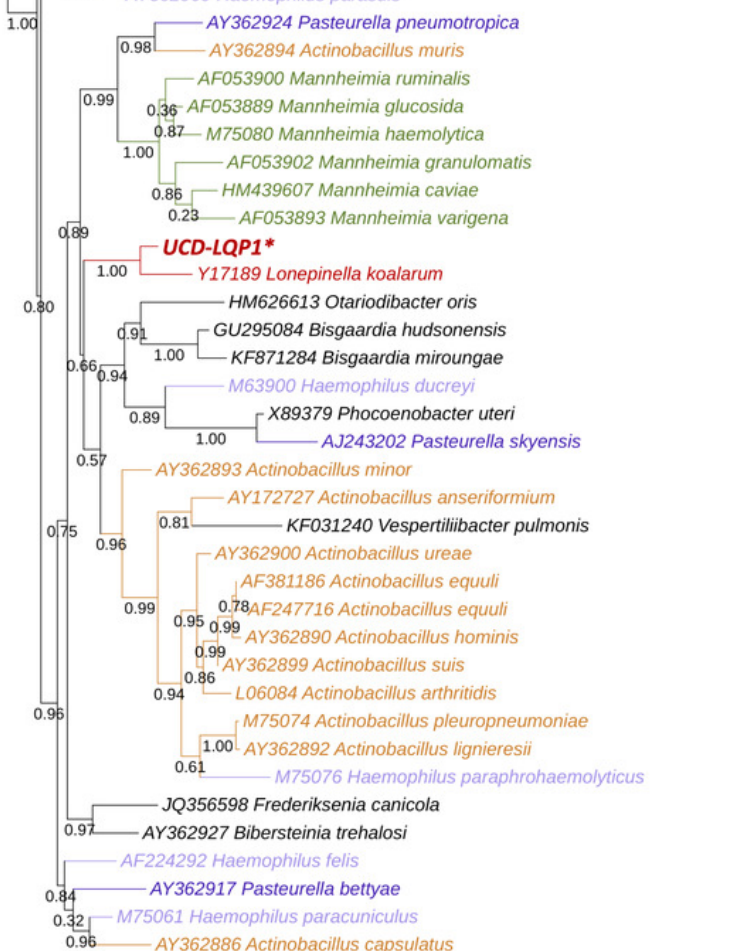




\section{Figure 2}

Pangenome comparison of $L$. koalarum strain UCD-LQP1 and 57 of its most closely related, publicly available genomes

This figure was generated from the microbial pangenomic analysis in Anvi'o version 5.5 where each ring represents an individual genome assembly. After ordering all taxa according to a genomic marker gene tree, genomes were colored following NCBI taxonomy (red: Lonepinella koalarum, dark purple: Pasteurella spp., light purple: Haemophilus spp., pink: Aggregatibacter spp., green: Mannheimia spp., light green: Avibacterium paragallinarum, grey: Necropsobacter spp., and yellow: Rodentibacter spp. Note, Actinobacillus spp. was colored in green here and not orange as in Fig. 1 to show its relation to Mannheimia spp. According to GTDB taxonomy, those two genomes are now Basfia species. See Discussion section). Each wedge represents a gene cluster. Gene clusters were grouped into mostly shared, shared, private, and in red: exclusively found in Lonepinella koalarum genome assemblies: 'LK', and exclusively found in L. koalarum strain UCD-LQP1. The gene marker tree was created in PhyloSift version 1.0.1 (Darling et al., 2014) with its updated markers database (version 4, posted on 12th of February 2018; Jospin, 2018) for the alignment and RAxML version 8.2.10 on the CIPRES web server for the tree inference (Miller, Pfeiffer \& Schwartz, 2010). Gene clusters were ordered based on presence/absence. Also shown is GC content in light brown, number of genes per kilo base pairs in light grey, number of gene clusters in dark grey, and number of singleton gene clusters in orange, for each assembly, respectively. The heatmap shows ANI (Average nucleotide identity) values $>95 \%$. The ANI heatmap is aligned with the Anvi'o profile, leading to the genome IDs on the y-axis. The Anvi'o database (Wilkins, 2020g) and profile (Wilkins, 2020h) are accessible on FigShare. 


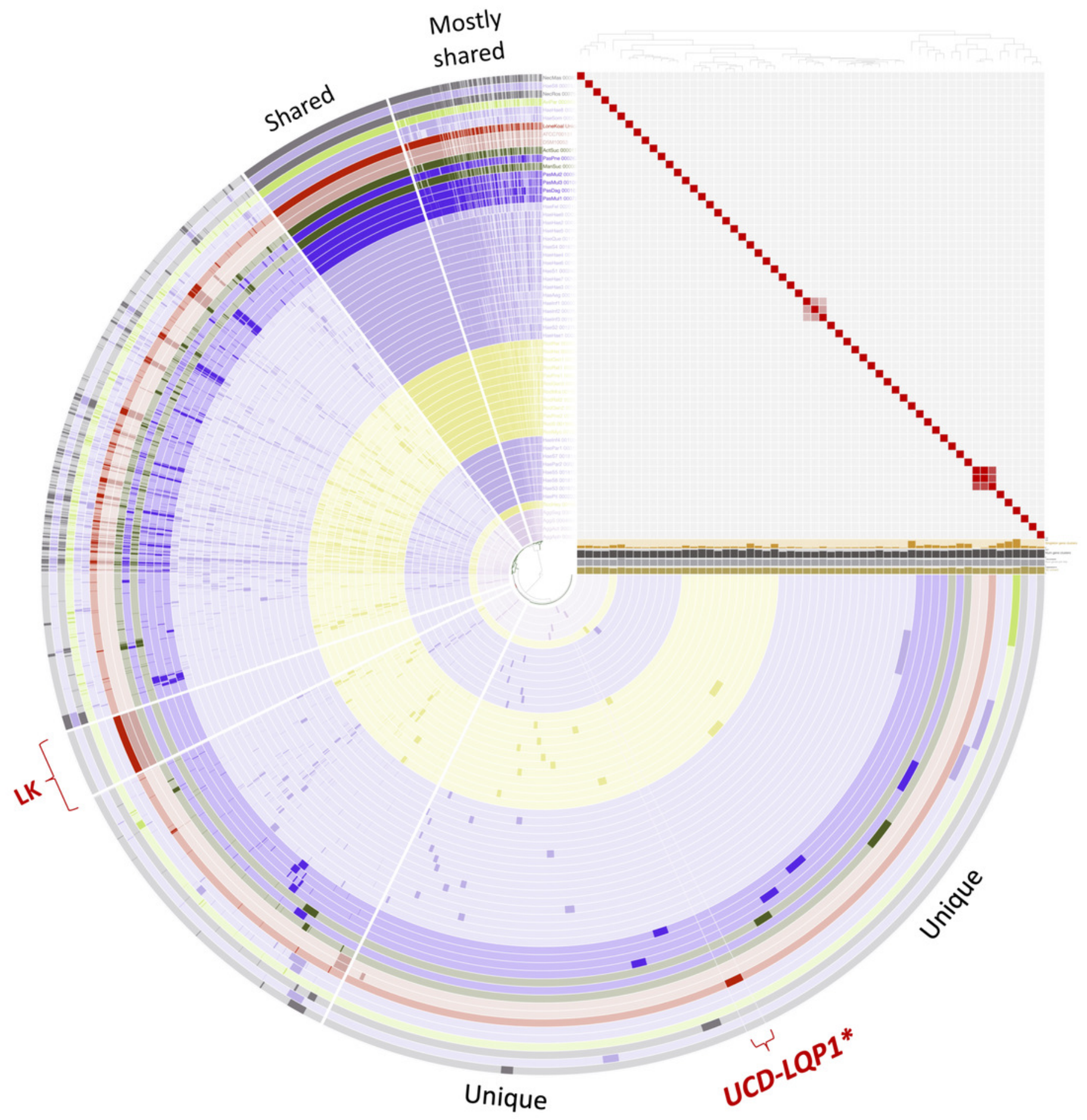




\section{Table $\mathbf{1}$ (on next page)}

Lonepinella koalarum strain UCD-LQP1 assembly statistics

Completeness and contamination were determined with CheckM version 1.0.8 (Parks et al., 2015); number of contigs, total length, GC\%, N50, N75, L50, and L75 were determined with QUAST (Quality Assessment Tool for Genome Assemblies) (Gurevich et al., 2013); number of predicted genes and number of protein coding genes were determined with PROKKA version 1.12 (Seemann, 2014). 
1

\begin{tabular}{ll}
\hline Statistic & Value \\
\hline Completeness & $99.205 \%$ \\
Contamination & $0.705 \%$ \\
Number of Contigs & 29 \\
Total Length & $2,608,483 \mathrm{bp}$ \\
GC\% & 39.02 \\
N50 & $2,299,135 \mathrm{bp}$ \\
N75 & $2,299,135 \mathrm{bp}$ \\
L50 & 1 \\
L75 & 1 \\
Number of Predicted Genes & 2,551 \\
Number of Protein Coding & 2,479 \\
Genes & \\
\hline
\end{tabular}

2 


\section{Table 2 (on next page)}

KEGG pathways involved in xenobiotics biodegradation and metabolism

Twenty KEGG pathways known to play a role in plant secondary metabolite degradation (Kanehisa \& Goto, 2000) were searched in the eggNOG annotations of Lonepinella koalarum strain UCD-LQP1 (Hits). The translated amino acid sequences encoded by putative genes in $L$. koalarum can be downloaded from FigShare (Wilkins, 2020b). 


\begin{tabular}{lll}
\hline KEGG: Xenobiotics biodegradation and metabolism & & \\
Pathway & Hits & KEGG ID \\
\hline Drug metabolism - Other enzymes & 15 & ko00983 \\
Benzoate degradation & 14 & ko00362 \\
Metabolism of xenobiotics by Cytochrome P450 & 9 & ko00980 \\
Chloroalkane and chloroalkene degradation & 8 & ko00625 \\
Aminobenzoate degradation & 6 & ko00627 \\
Xylene degradation & 6 & ko00622 \\
Naphthalene degradation & 6 & ko00626 \\
Dioxin degradation & 5 & ko00621 \\
Chlorocyclohexane and chlorobenzene degradation & 3 & ko00361 \\
Toluene degradation & 2 & ko00623 \\
Nitrotoluene degradation & 2 & ko00633 \\
Styrene degradation & 2 & ko00643 \\
Fluorobenzoate degradation & 1 & ko00364 \\
Ethylbenzene degradation & 0 & ko00642 \\
Atrazine degradation & 0 & ko00791 \\
Caprolactam degradation & 0 & ko00930 \\
Bisphenol degradation & 0 & ko00363 \\
Polycyclic aromatic hydrocarbon degradation & 0 & ko00624 \\
Furfural degradation & 0 & ko00365 \\
Steroid degradation & 0 & ko00984 \\
\hline
\end{tabular}

1 


\section{Table 3 (on next page)}

Unique gene clusters and their COG IDs in three Lonepinella koalarum genome assemblies

A comparative genomic analysis was performed in Anvi'o version 5.5 (Eren et al., 2015) to compare three publicly available Lonepinella koalarum genome assemblies to 55 closely related genomes. Gene clusters that could only be found in $L$. koalarum relative to the rest were filtered out and annotated with COG (Clusters of Orthologous Groups). Carbohydrateactive enzymes are shaded in grey. Only a subset of COG categories are shown; G:

'Carbohydrate metabolism/transport', M: 'Cell wall', H: 'Coenzyme metabolism', V: 'Defense',

C: 'Energy production', W: 'Extracellular', P: 'Inorganic ion transport', X: 'Prophages and Transposon', L: 'Replication and Repair'. The complete list of unique gene clusters and their translated amino acid sequence in L. koalarum can be found in Supplementary Tables S4-S6. 


\begin{tabular}{|c|c|c|c|}
\hline $\begin{array}{c}\text { Gene } \\
\text { ID }\end{array}$ & COG ID & COG Function & $\begin{array}{c}\text { COG } \\
\text { Category }\end{array}$ \\
\hline 1408 & COG1501 & Alpha-glucosidase, glycosyl hydrolase family GH31 & G \\
\hline 1251 & COG3534 & Alpha-L-arabinofuranosidase|Alpha-L-arabinofuranosidase & G \\
\hline 1643 & COG2723 & Beta-galactosidase & G \\
\hline 1513 & COG0129 & $\begin{array}{l}\text { Dihydroxyacid dehydratase/phosphogluconate dehydratase } \\
\text { DNA-binding transcriptional regulator of sugar metabolism, DeoR/GlpR }\end{array}$ & G \\
\hline 1404 & COG1349 & family & G \\
\hline 1410 & COG2017 & Galactose mutarotase or related enzyme & G \\
\hline 1204 & COG2220 & L-ascorbate metabolism protein UlaG, beta-lactamase superfamily & G \\
\hline 1409 & COG2942 & Mannose or cellobiose epimerase, $\mathrm{N}$-acyl-D-glucosamine 2-epimerase family & G \\
\hline 1250 & COG2211 & $\mathrm{Na}+/$ melibiose symporter or related transporter & G \\
\hline 1642 & COG1472 & Periplasmic beta-glucosidase and related glycosidases & G \\
\hline 810 & COG1447 & Phosphotransferase system cellobiose-specific component IIA & G \\
\hline 812 & COG1440 & Phosphotransferase system cellobiose-specific component IIB & G \\
\hline 808 & COG1455 & Phosphotransferase system cellobiose-specific component IIC & G \\
\hline 2231 & COG1263 & Phosphotransferase system IIC components, glucose-specific & $\mathrm{G}$ \\
\hline 811 & COG1762 & Phosphotransferase system mannitol/fructose-specific IIA domain (Ntr-type) & $\mathrm{G}$ \\
\hline 2230 & COG1621 & Sucrose-6-phosphate hydrolase SacC, GH32 family & G \\
\hline 1372 & COG0524 & Sugar or nucleoside kinase, ribokinase family & G \\
\hline 1407 & COG3684 & Tagatose-1,6-bisphosphate aldolase & G \\
\hline 1729 & COG3711 & $\begin{array}{l}\text { Transcriptional antiterminator|Mannitol/fructose-specific phosphotransferase } \\
\text { system, IIA domain }\end{array}$ & G \\
\hline 1402 & COG1593 & TRAP-type C4-dicarboxylate transport system, large permease component & G \\
\hline 1401 & COG1638 & TRAP-type C4-dicarboxylate transport system, periplasmic component & G \\
\hline 1403 & COG3090 & TRAP-type C4-dicarboxylate transport system, small permease component & G \\
\hline 1023 & COG0859 & ADP-heptose:LPS heptosyltransferase & M \\
\hline 815 & COG3659 & Carbohydrate-selective porin OprB & M \\
\hline 264 & COG3765 & LPS O-antigen chain length determinant protein, WzzB/FepE family & M \\
\hline 1623 & COG1388 & LysM repeat & M \\
\hline 2084 & COG2244 & Membrane protein involved in the export of O-antigen and teichoic acid & M \\
\hline 489 & COG0451 & Nucleoside-diphosphate-sugar epimerase & M \\
\hline 1468 & COG3307 & O-antigen ligase & M \\
\hline 221 & COG3203 & Outer membrane protein (porin) & M \\
\hline 557 & COG1538 & Outer membrane protein TolC & M \\
\hline 199 & COG0810 & Periplasmic protein TonB, links inner and outer membranes & M \\
\hline 1187 & COG2843 & $\begin{array}{l}\text { Poly-gamma-glutamate biosynthesis protein CapA/YwtB (capsule formation), } \\
\text { metallophosphatase superfamily }\end{array}$ & M \\
\hline 495 & COG0043 & 3-polyprenyl-4-hydroxybenzoate decarboxylase & $\mathrm{H}$ \\
\hline 1371 & COG3201 & Nicotinamide riboside transporter PnuC & $\mathrm{H}$ \\
\hline 413 & COG4206 & Outer membrane cobalamin receptor protein & $\mathrm{H}$ \\
\hline 817 & COG1477 & Thiamine biosynthesis lipoprotein ApbE & $\mathrm{H}$ \\
\hline 2228 & COG2226 & Ubiquinone/menaquinone biosynthesis C-methylase UbiE & $\mathrm{H}$ \\
\hline 2071 & COG1401 & $\begin{array}{l}\text { 5-methylcytosine-specific restriction endonuclease McrBC, GTP-binding } \\
\text { regulatory subunit McrB }\end{array}$ & $\mathrm{V}$ \\
\hline 1160 & COG1132 & ABC-type multidrug transport system, ATPase and permease component & $\mathrm{V}$ \\
\hline 1058 & COG4823 & Abortive infection bacteriophage resistance protein & $\mathrm{V}$ \\
\hline 1573 & COG0251 & Enamine deaminase RidA, house cleaning of reactive enamine intermediates & $\mathrm{V}$ \\
\hline
\end{tabular}




\begin{tabular}{|c|c|c|c|}
\hline 1669 & COG2337 & $\begin{array}{l}\text { mRNA-degrading endonuclease, toxin component of the MazEF toxin- } \\
\text { antitoxin module }\end{array}$ & $\mathrm{V}$ \\
\hline 2012 & COG0845 & Multidrug efflux pump subunit AcrA (membrane-fusion protein) & $\mathrm{V}$ \\
\hline 432 & COG3093 & $\begin{array}{l}\text { Plasmid maintenance system antidote protein VapI, contains XRE-type HTH } \\
\text { domain }\end{array}$ & $\mathrm{V}$ \\
\hline 1138 & COG2828 & 2-Methylaconitate cis-trans-isomerase $\operatorname{PrpF}$ (2-methyl citrate pathway) & $\mathrm{C}$ \\
\hline 9 & COG1048 & Aconitase A & $\mathrm{C}$ \\
\hline 311 & COG1454 & Alcohol dehydrogenase, class IV & $\mathrm{C}$ \\
\hline 1134 & COG3312 & FoF1-type ATP synthase assembly protein I & $\mathrm{C}$ \\
\hline 2130 & COG0435 & Glutathionyl-hydroquinone reductase & $\mathrm{C}$ \\
\hline 763 & COG0371 & Glycerol dehydrogenase or related enzyme, iron-containing ADH family & $\mathrm{C}$ \\
\hline 1584 & COG0778 & Nitroreductase & $\mathrm{C}$ \\
\hline 816 & COG1053 & Succinate dehydrogenase/fumarate reductase, flavoprotein subunit & $\mathrm{C}$ \\
\hline 514 & COG4972 & Tfp pilus assembly protein, ATPase PilM & $\mathrm{W}$ \\
\hline 2098 & COG1116 & $\begin{array}{l}\text { ABC-type nitrate/sulfonate/bicarbonate transport system, ATPase component } \\
\text { ABC-type nitrate/sulfonate/bicarbonate transport system, periplasmic }\end{array}$ & $\mathrm{P}$ \\
\hline 2097 & COG0715 & component & $\mathrm{P}$ \\
\hline 592 & COG0600 & ABC-type nitrate/sulfonate/bicarbonate transport system, permease component & $\mathrm{P}$ \\
\hline 818 & COG2807 & Cyanate permease & $\mathrm{P}$ \\
\hline 2005 & COG2382 & Enterochelin esterase or related enzyme & $\mathrm{P}$ \\
\hline 234 & COG3301 & Formate-dependent nitrite reductase, membrane component $\mathrm{NrfD}$ & $\mathrm{P}$ \\
\hline 365 & COG3230 & Heme oxygenase & $\mathrm{P}$ \\
\hline 1053 & COG0672 & $\begin{array}{l}\text { High-affinity } \mathrm{Fe} 2+/ \mathrm{Pb} 2+\text { permease } \\
\text { Iron uptake system EfeUOB, periplasmic (or lipoprotein) component }\end{array}$ & $\mathrm{P}$ \\
\hline 1051 & COG2822 & $\begin{array}{l}\text { EfeO/EfeM } \\
\text { NADPH-dependent ferric siderophore reductase, contains FAD-binding and }\end{array}$ & $\mathrm{P}$ \\
\hline 28 & COG2375 & SIP domains & $\mathrm{P}$ \\
\hline 1158 & COG2223 & Nitrate/nitrite transporter NarK & $\mathrm{P}$ \\
\hline 1209 & COG2223 & Nitrate/nitrite transporter NarK & $\mathrm{P}$ \\
\hline 1089 & COG4771 & Outer membrane receptor for ferrienterochelin and colicins & $\mathrm{P}$ \\
\hline 1052 & COG2837 & Periplasmic deferrochelatase/peroxidase EfeB & $\mathrm{P}$ \\
\hline 310 & COG0659 & Sulfate permease or related transporter, MFS superfamily & $\mathrm{P}$ \\
\hline 631 & COG4388 & Mu-like prophage I protein & $\mathrm{X}$ \\
\hline 662 & COG2932 & $\begin{array}{l}\text { Phage repressor protein C, contains Cro/C1-type HTH and peptisase s } 24 \\
\text { domains }\end{array}$ & $\mathrm{X}$ \\
\hline 2183 & COG5412 & Phage-related protein & $\mathrm{X}$ \\
\hline 1780 & COG1943 & REP element-mobilizing transposase RayT & $\mathrm{X}$ \\
\hline 1057 & COG2189 & Adenine specific DNA methylase Mod & $\mathrm{L}$ \\
\hline 48 & COG1074 & $\begin{array}{l}\text { ATP-dependent exoDNAse (exonuclease V) beta subunit (contains helicase } \\
\text { and exonuclease domains) } \\
\text { ATP-dependent exoDNAse (exonuclease V), alpha subunit, helicase }\end{array}$ & $\mathrm{L}$ \\
\hline 47 & COG0507 & superfamily I & $\mathrm{L}$ \\
\hline 2035 & COG3057 & Negative regulator of replication initiation & $\mathrm{L}$ \\
\hline
\end{tabular}




\section{Table 4 (on next page)}

Putative carbohydrate-active enzymes (CAZy) found in Lonepinella koalarum genome assemblies

Genes coding for putative carbohydrate-active enzymes (CAZy enzymes) that were only found in Lonepinella koalarum relative to 55 closely related genomes are shaded in grey (corresponding to Table 3 ), and CAZy enzymes that were only found in the assembly of $L$. koalarum UCD-LQP1 alone are presented in bold. The position of genes potentially coding for these enzymes in the assembly of L. koalarum UCD-LQP1 are shown as well. See Material and methods section for details on how assemblies were screened for CAZy enzymes. Positions of these genes in L. koalarum genome assemblies ATCC 700131 and DSM 10053 are shown in Supplementary Table S7. 


\begin{tabular}{|c|c|c|}
\hline CAZy & Enzyme & $\begin{array}{c}\text { LK } \\
\text { position }\end{array}$ \\
\hline GH1 & $\beta$-Glycosidase; membrane-bound lytic transglycosylase A (MltA) & $1 \_1622$ \\
\hline $\mathrm{GH} 2$ & $\beta$-Galactosidase & $1 \_1329$ \\
\hline GH3 & Glycoside hydrolase Family 3 & $1 \_1536$ \\
\hline GH4 & $\begin{array}{l}\alpha \text { - and } \beta \text {-Glycosidases } \\
\text { Major glycoside hydrolase family acting on substrates containing } \alpha \text {-glucoside }\end{array}$ & $1 \_1355$ \\
\hline GH13 & linkages & $1 \_1263$ \\
\hline GH20 & Retaining glycoside hydrolases & 1_757 \\
\hline $\mathrm{GH} 23$ & Lytic transglycosylases of $\mathrm{GH} 23$ & $1 \_1073$ \\
\hline GH31 & $\alpha$-Glucosidases & $1 \_1388$ \\
\hline GH32 & Inverting sucrose; invertase & $2 \_66$ \\
\hline GH33 & Glycoside hydrolase family 33 & $1 \_1214$ \\
\hline GH42 & Plant cell wall degradation & 1_1367 \\
\hline GH43 & $\alpha$-L-Arabinofuranosidase and $\beta$-D-xylosidase activity & $1 \_1369$ \\
\hline GH77 & $\alpha$-Amylase & 38 \\
\hline GH102 & Lytic transglycosylases & $1 \_346$ \\
\hline GH103 & Lytic transglycosylase B (MltB) & $2 \_81$ \\
\hline CE4 & Deacylation of polysaccharides & 1_760 \\
\hline CE9 & Deacetylation of N-acetylglucosamine-6-phosphate & $1 \_1583$ \\
\hline CE11 & Carbohydrate esterase family 11 & $1 \_1738$ \\
\hline GT2 & Glycosyltransferase family 2 & $1 \_759$ \\
\hline GT5 & Glycosyltransferase family 5 & 3_4 \\
\hline GT9 & Glycosyltransferase family 9 & $1 \_999$ \\
\hline GT19 & Glycosyltransferase family 19 & 1_1807 \\
\hline GT28 & $\beta-1,4-G l c N A c$ Transferase & 1_1732 \\
\hline GT30 & Glycosyltransferase family 30 & $1 \_855$ \\
\hline GT35 & Glycogen and starch phosphorylase & $1 \_1193$ \\
\hline GT41 & N-glycosyltransferase & $1 \_943$ \\
\hline GT51 & Murein polymerase & 271 \\
\hline
\end{tabular}

